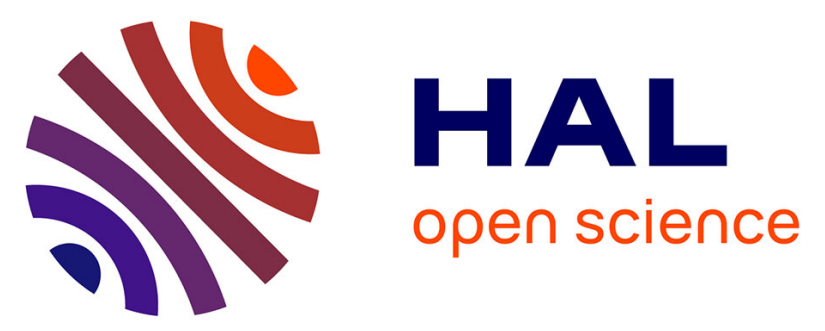

\title{
Reduced RNA turnover as a driver of cellular senescence
}

Nowsheen Mullani, Yevheniia Porozan, Adèle Mangelinck, Christophe Rachez, Mickaël Costallat, Eric Batsche, Michele Goodhardt, Giovanni Cenci, Carl Mann, Christian Muchardt

\section{- To cite this version:}

Nowsheen Mullani, Yevheniia Porozan, Adèle Mangelinck, Christophe Rachez, Mickaël Costallat, et al. Reduced RNA turnover as a driver of cellular senescence. Life Science Alliance, 2021, 4 (3), pp.e202000809. 10.1101/800128 . hal-02324027v2

\section{HAL Id: hal-02324027 \\ https://hal.science/hal-02324027v2}

Submitted on 26 Jan 2021

HAL is a multi-disciplinary open access archive for the deposit and dissemination of scientific research documents, whether they are published or not. The documents may come from teaching and research institutions in France or abroad, or from public or private research centers.
L'archive ouverte pluridisciplinaire HAL, est destinée au dépôt et à la diffusion de documents scientifiques de niveau recherche, publiés ou non, émanant des établissements d'enseignement et de recherche français ou étrangers, des laboratoires publics ou privés. 


\title{
Reduced RNA turnover as a driver of cellular senescence
}

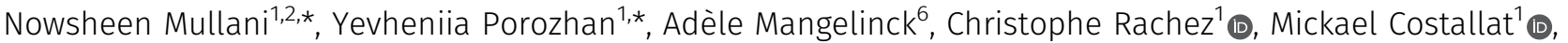

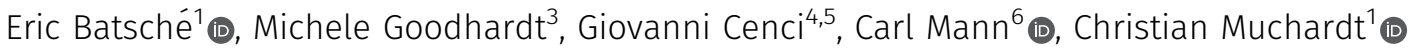

\begin{abstract}
Accumulation of senescent cells is an important contributor to chronic inflammation upon aging. The inflammatory phenotype of senescent cells was previously shown to be driven by cytoplasmic DNA. Here, we propose that cytoplasmic double-stranded RNA has a similar effect. We find that several cell types driven into senescence by different routes share an accumulation of long promoter RNAs and $3^{\prime}$ gene extensions rich in retrotransposon sequences. Accordingly, these cells display increased expression of genes involved in response to double stranded RNA of viral origin downstream of the interferon pathway. The RNA accumulation is associated with evidence of reduced RNA turnover, including in some cases, reduced expression of RNA exosome subunits. Reciprocally, depletion of RNA exosome subunit EXOSC3 accelerated expression of multiple senescence markers. A senescence-like RNA accumulation was also observed in cells exposed to oxidative stress, an important trigger of cellular senescence. Altogether, we propose that in a subset of senescent cells, repeat-containing transcripts stabilized by oxidative stress or reduced RNA exosome activity participate in driving and maintaining the permanent inflammatory state characterizing cellular senescence.
\end{abstract}

DOI 10.26508/lsa.202000809 | Received 8 June 2020 | Revised 22 December 2020 | Accepted 23 December 2020 | Published online 14 January 2021

\section{Introduction}

Cellular senescence is a state of irreversible cell cycle arrest (Rodier \& Campisi, 2011). Experimentally, it can be triggered via multiple routes including prolonged maintenance in tissue culture, exposure to ionizing radiations or oxidative stress, and forced expression of mitogenic oncogenes. These inducers of senescence all share the ability to cause DNA damage and to generate reactive oxygen species, two components that probably are at the basis of the phenomenon (Ben-Porath \& Weinberg, 2005). One of the hallmarks of senescent cells is their production of a range of chemokines, pro-inflammatory cytokines, growth factors, and matrixremodeling enzymes, defining the senescence-associated secretory phenotype or SASP. This pro-inflammatory characteristic has a crucial role in propagating senescence and in recruiting immune cells to the senescent tissue. As senescent cells accumulate with time, the SASP is also believed to be a major determinant of the chronic low-grade inflammation associated with aging and agerelated diseases.

SASP activation is largely orchestrated by NF-kB and CCAAT/ enhancer-binding protein beta (C/EBPb). Upstream of these transcription factors, DNA damage and the DNA damage response are major triggers of the pro-inflammatory pathways (Salminen et al, 2012). Yet, it seems that other mechanisms may allow nucleic acids to drive the chronic sterile inflammation characteristic of cellular senescence. Indeed, several studies have associated senescence with an accumulation of DNA in the cytoplasm. This DNA, in the form of chromatin, triggers innate immunity via the cytosolic DNA-sensing CGAS-STING pathway (Dou et al, 2017). Cytoplasmic DNA possibly originates from chromosome segregation errors during mitosis and its accumulation seems favored by downregulation in senescent cells of the cytoplasmic DNases TREX1 and DNASE2 (Takahashi et al, 2018). In addition, it has been shown that in senescent cells, de-repression of repeat elements of the LINE family results in the production of retroviral RNAs, which after retrotranscription, accumulate in the cytoplasm in the form of cDNAs (Cecco et al, 2019).

Consistent with a role of cytoplasmic DNA, multiple studies have documented the importance of the interferon pathway in driving senescence, and suppression of type 1 interferon signaling hinders the onset of senescence (Katlinskaya et al, 2016). The interferon pathway was initially described as an antiviral defense mechanism activated by specific cytoplasmic or endosomal receptors of either viral DNA or dsRNA (Colby \& Morgan, 1971). In this context, RNA could also be a trigger of senescence.

\footnotetext{
'Institut Pasteur, Centre National de la Recherche Scientifique (CNRS) UMR3738, Dpt Biologie du Développement et Cellules Souches, Unité de Régulation Epigénétique Paris, France 'Sorbonne Université, Ecole Doctorale "Complexité du Vivant" (ED515), Paris, France ${ }^{3}$ Institut National de la Santé et de la Recherche Médicale (INSERM) U976, Institut de Recherche Saint Louis, Université de Paris, Paris, France ${ }^{4}$ Dipartimento Biologia e Biotecnologie “C. Darwin," SAPIENZA Università di Roma, Rome, Italy ${ }^{5}$ Istituto Pasteur Italia-Fondazione Cenci Bolognetti, Rome, Italy ${ }^{6}$ Université Paris-Saclay, Commissariat à l'Énergie Atomique et aux Énergies Alternatives (CEA), Centre National de la Recherche Scientifique (CNRS), Institute for Integrative Biology of the Cell (I2BC), Gif-sur-Yvette, France
}

Correspondence: christian.muchardt@sorbonne-universite.fr

Christophe Rachez, Eric Batsché, and Christian Muchardt's present address is Institut de Biologie Paris-Seine (IBPS), Institut National de la Recherche Scientifique (CNRS) UMR8256, Paris, France

*Nowsheen Mullani and Yevheniia Porozhan contributed equally to this work 
To date, the role of RNA in senescence has mostly been examined at the level of discrete long non-coding RNAs (IncRNAs) regulating expression or activity of proteins relevant for cellular senescence (Montes \& Lund, 2016). For example, VAD, a "very IncRNA," partially antisense to the DDAH1 gene, inhibits the incorporation of the repressive histone variant H2A.Z at the promoter of the INK4 gene in senescent cells (Lazorthes et al, 2015). Inversely, SENEBLOC, a Myc-regulated IncRNA, interferes with senescence in part by regulating $\mathrm{p53}$-mediated repression of the $\mathrm{p} 21$ gene $(\mathrm{Xu}$ et $\mathrm{al}, 2020$ ). The InCRNAs HOTAIR and MALAT were also reported as regulated during entry into senescence (Tripathi et al, 2013; Yoon et al, 2013). However, some observations are suggestive of an impact of RNAs in cellular senescence at other levels, via their production, their maturation, or their turnover. For example, it was reported that neurons in the aging mouse brain accumulate 3' UTRs, resulting in the production of small peptides of yet unknown function (Sudmant et al, 2018). Furthermore, transcripts from SINEs/Alus, a family of repetitive elements particularly abundant in euchromatin, were reported to accumulate in senescent cells with an impact on genome integrity (Wang et al, 2014). Finally, several studies have reported that cellular senescence is associated with numerous changes in the outcome of alternative pre-mRNA splicing (Wang et al, 2018). This results in the production of senescent toxins, including progerin, a variant of Lamin A associated with the Hutchinson-Gilford progeria syndrome (Cao et al, 2011), while also favoring the synthesis of S-Endoglin and p53 $\beta$ that may have similar pro-senescence activities (Fujita et al, 2009; Miao et al, 2016).

Here, to focus on a possible role for RNA in the triggering of the interferon pathway during the onset of senescence, we examined different senescent cells for sources of RNA species liable to activate innate immunity. This approach revealed that in several cell lines, senescence is associated with a gradual accumulation of reads originating from regions located upstream or downstream of genes and extending outside of the transposon-free regions characterizing transcription start and termination sites. Accumulation of these RNAs was correlated with reduced RNA turnover, accumulation of dsRNA in the cytoplasm, and increased activity of antiviral pathways dedicated to the degradation of dsRNAs. RNA turnover was not affected in growth-arrested cells. Consistent with a pro-senescence activity of the accumulating RNAs, inactivation of RNA exosome activity caused premature expression of senescence markers in human fibroblasts challenged with oncogenic RAF. Finally, we noted that oxidative stress caused accumulation of RNA species largely resembling those observed in senescent cells, suggesting that these RNAs participate in linking mitochondrial suffering to the permanent inflammatory state associated with senescence.

\section{Results}

\section{Accumulation of RNA exosome substrates in senescent Wi38 cells}

It was previously reported that in Wi38 cells driven into senescence by tamoxifen-inducible oncogenic RAF, many convergent genes displayed transcriptional termination read-through resulting in transcriptional interference (Muniz et al, 2017). In the RNA-seq data from this study, the read-through translated into an accumulation of sequencing reads downstream of several convergent genes in the senescent cells. Re-examination of these data revealed that downstream reads accumulated at a wide range of genes beyond those described in the initial study. For example, we observed accumulation of downstream reads at several canonical histone genes (e.g., Figs $1 \mathrm{~A}$ and S1A, green arrows). As these genes are expressed mainly in the $S$ phase of the cell cycle and therefore strongly down-regulated in senescent cells, accumulation of these reads appeared uncoupled from the activity of the cognate gene (compare the two different scales in Figs 1A and S1A).

In the RNA-seq data, we also noted accumulation of reads downstream of several non-coding RNAs (ncRNAs). In particular, we found increased levels of $3^{\prime}$ extensions at essentially all $U$ small nuclear RNA (snRNA) genes expressed in the Wi38 cells (e.g., Figs 1B and S1B, and heat map in Fig 1C). Upstream antisense RNAs (uaRNAs, also known as PROMPTs), another category of ncRNAs resulting from bi-directional initiation at promoters (schematic Fig 1D) were also accumulating at numerous genes (e.g., Fig $1 \mathrm{~A}$ and $\mathrm{E}$, blue arrows). In total, quantification at the series of 5,260 promoters not overlapping with coding regions of any gene revealed an $\sim 10 \%$ increase in uaRNA accumulation in the senescent cells (Fig 1F and $G$ ).

Together, these observations revealed that oncogene-induced senescence in Wi38 cells is associated with an accumulation of transcripts originating from regions upstream and downstream of genes. We will refer to these RNAs as perigenic RNAs (pegeRNAs).

\section{Modified RNA catabolism in senescent Wi38 cells}

Examination of publicly available data from HeLa cells depleted of EXOSC3 (Schlackow et al, 2017) revealed that the RNA species accumulating in senescent Wi38 cells were strikingly similar to those accumulating upon inactivation of the RNA exosome (Fig S2A-C). We therefore examined RNA exosome subunit expression in the available transcriptomic data from proliferating and senescent Wi38 cells. This allowed us to identify a strong down-regulation in the senescent cells, of DIS3L, encoding the catalytic subunit of the cytoplasmic RNA exosome complex. This decreased expression was confirmed by quantitative RT PCR (Fig 2A). Western blotting further revealed decreased accumulation of the DIS3L protein in the senescent cells, whereas levels of the senescence marker CDKN1A/ p21 were increased (Fig 2B). We also examined the transcriptomes for signs of activation of defense mechanisms against cytoplasmic RNA. This revealed increased expression of OASL and NLRP3, both confirmed by quantitative RT PCR (Fig 2C). OASL is associated with the antiviral OAS/RNASEL pathway detecting dsRNAs (Silverman, 2007), whereas NLRP3 is the sensor component of the NLRP3 inflammosome, functioning as a dsRNA receptor (Franchi et al, 2014).

To gain further evidence for a reduced RNA turnover in the senescent Wi38 cells, we compared accumulation of short-lived and long-lived mRNAs, the assumption being that unstable RNAs would be more affected by fluctuation in RNA exosome activity than would the stable mRNAs. For this, we established a list of highly unstable $(\mathrm{t} 1 / 2<2 \mathrm{~h})$ or highly stable $(\mathrm{t} 1 / 2>10 \mathrm{~h})$ mRNAs from a 


\section{A chr6:26,190,280-26,208,795}

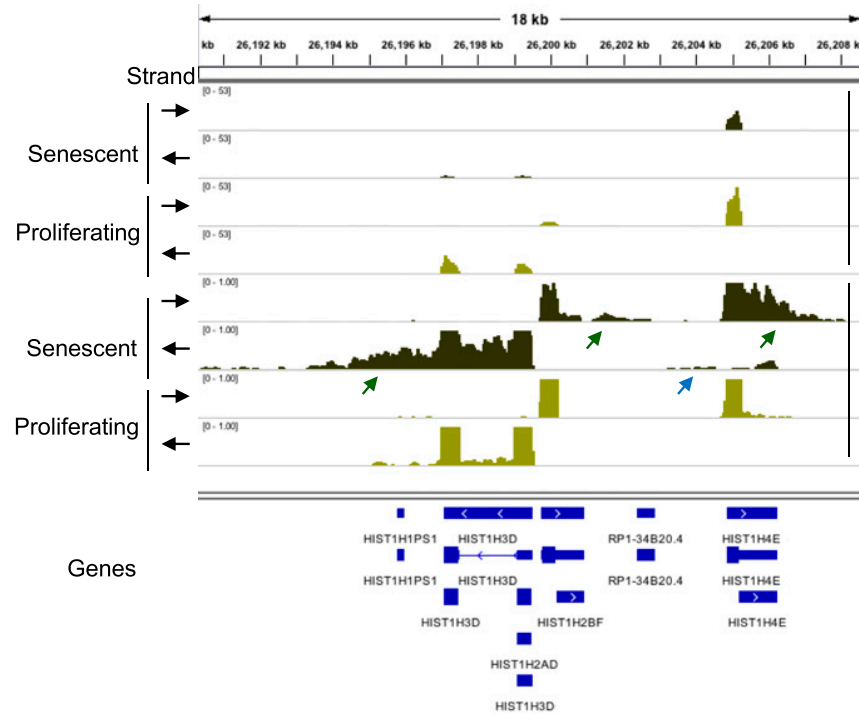

D

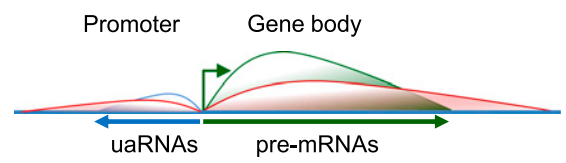

B chr1:11,968,083-11,970,125

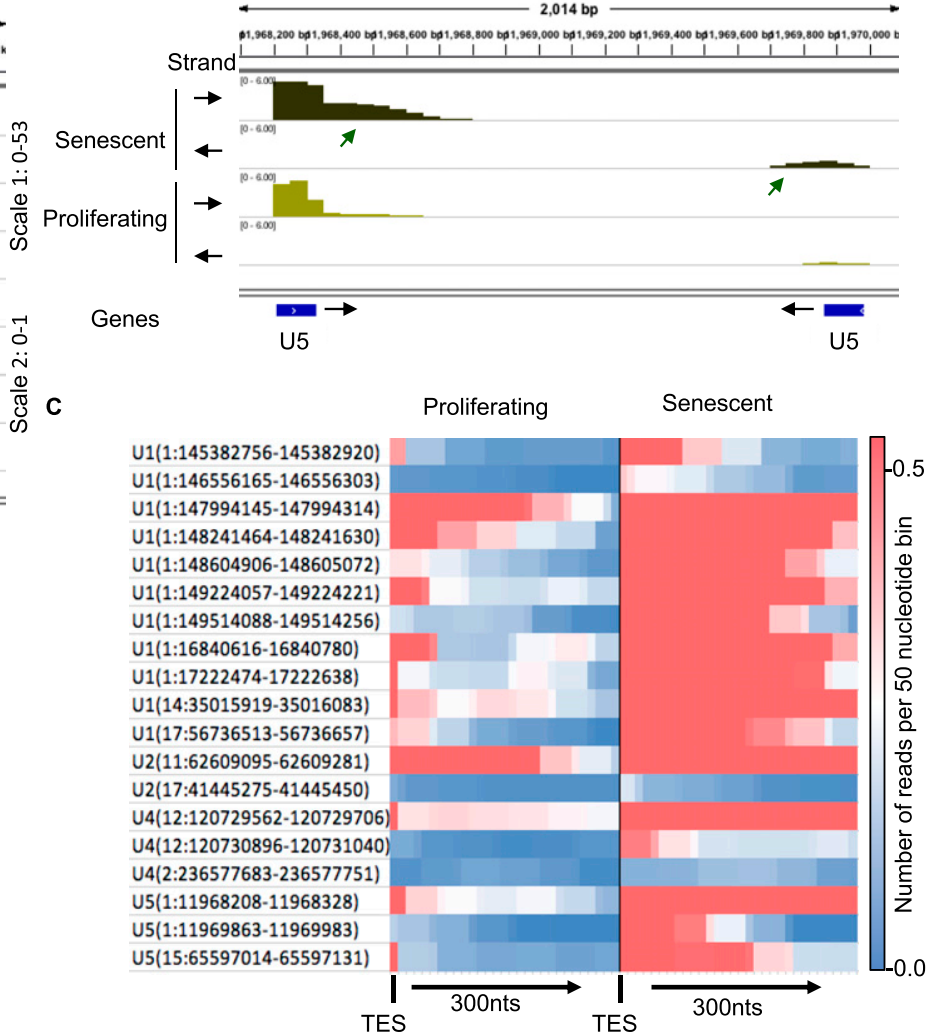

$$
\text { G }
$$

meta-promoter region in Wi38 cells

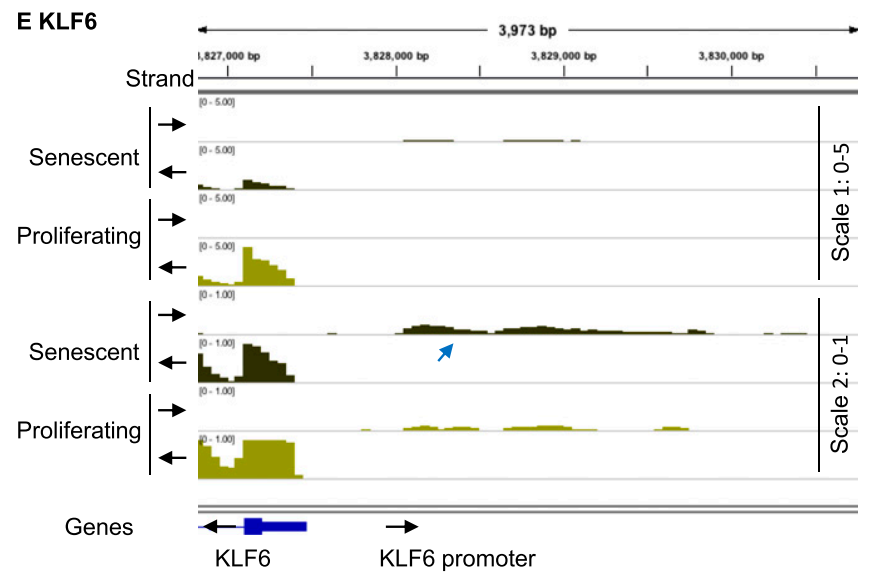

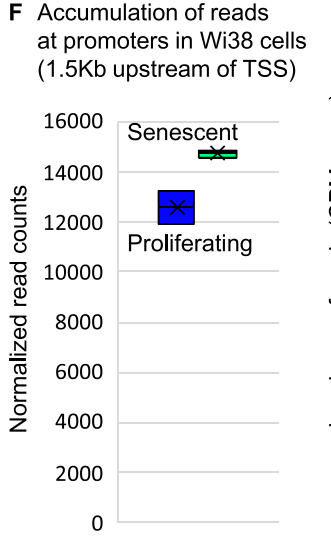

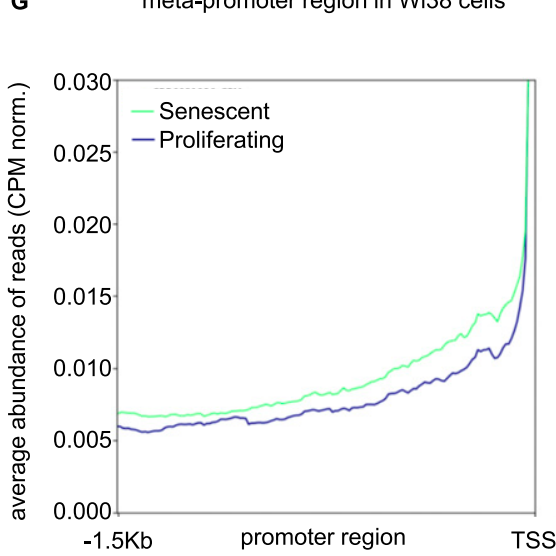

Figure 1. Senescent WI38 cells accumulate pegeRNAs.

RNA-seq data from WI38 hTERT RAF1-ER human fibroblasts either proliferating or driven into senescence by induction of RAF1-ER (Muniz et al, 2017). (A, B, E) Indicated loci were visualized with Integrative Genomics Viewer. Black arrows indicate the orientation of the track. Green arrows indicate 3' extensions, blue arrows, promoter RNAs. (C) Heat map illustrating increased accumulation of 3' extensions of U snRNAs in senescent versus proliferating WI38 hTERT RAF1-ER cells. Transcription end site indicates the $3^{\prime}$ end of the $U$ snRNA gene. (D) Schematic representation of divergent transcription at promoters. Green line represents pre-mRNA, blue line, normal accumulation of upstream antisense RNAs, red line, accumulation of upstream antisense RNAs in senescent cells. (F) At 5,260 promoters not overlapping with coding regions of any gene, reads were counted within a region of 1,500 nucleotides upstream of the transcription start site (TSS) in either proliferating or senescent WI38 hTERT RAF1-ER cells. (G) Average profile of read distribution along the 5,260 promoters in proliferating and senescent WI38 hTERT RAF1-ER cells.

genome-wide study on mRNA stability (Tani et al, 2012). These mRNAs are listed in Table S1. We first verified our assumption by examining the effect of reduced RNA exosome activity on accumulation of these mRNAs in the HeLa cell data. This showed that depletion of EXOSC3 resulted in increased accumulation of the short-lived RNAs when compared with WT cells. In contrast, levels of long-lived mRNAs appeared moderately decreased (Fig S2C and D). We explain this decrease by the augmented complexity of the 
A
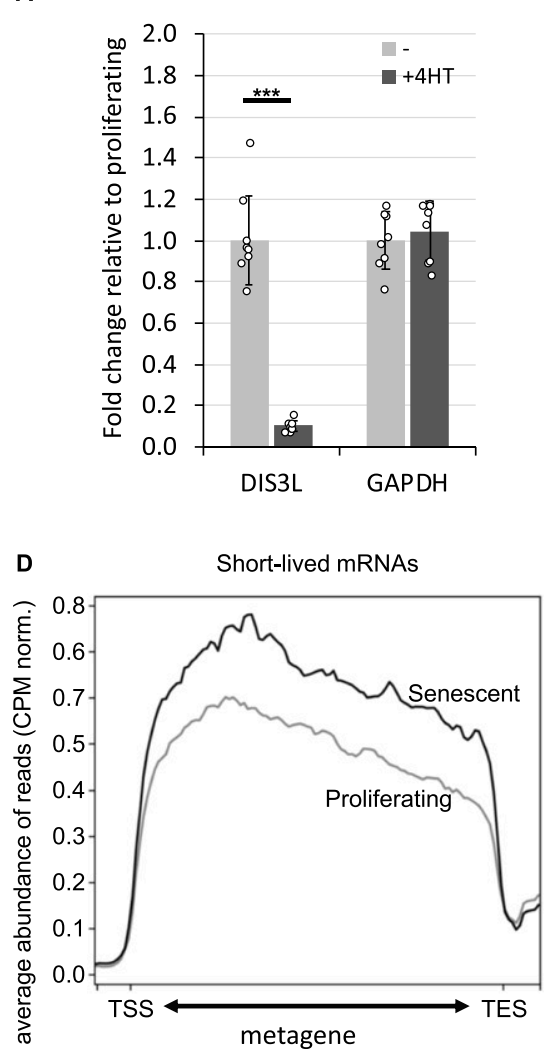

B

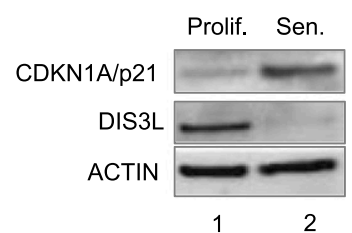

$\mathbf{E}$

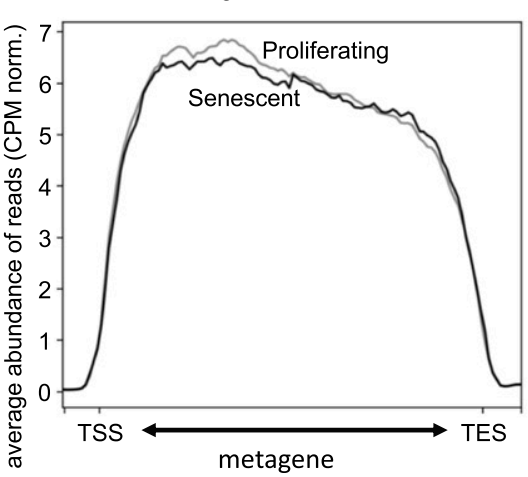

C

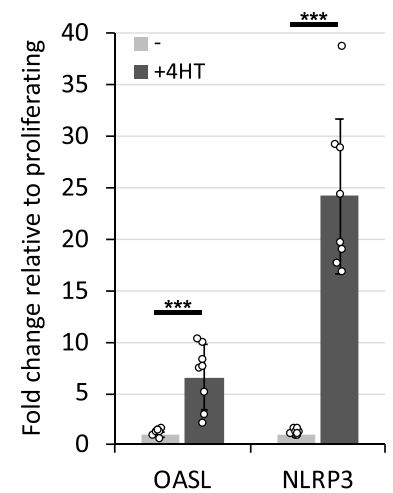

$\mathbf{F}$

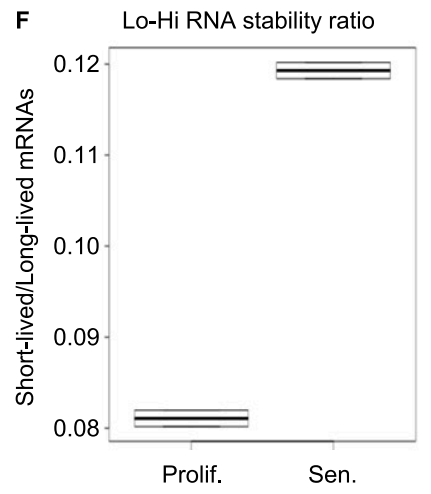

G

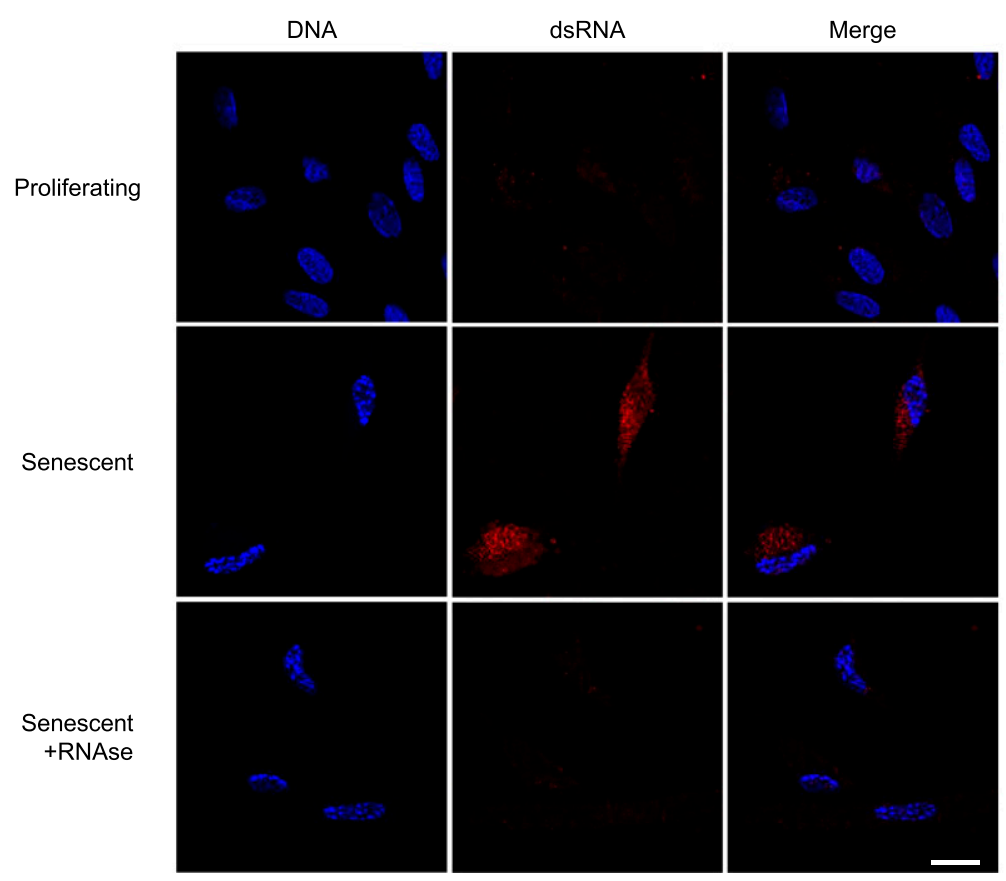

Figure 2. Reduced expression of RNA exosome subunits in senescence.

$(\mathbf{A}, \mathbf{B}, \mathbf{C})$ Total RNA or protein extracts were prepared from WI38 hTERT RAF1-ER human fibroblasts either proliferating or driven into senescence by induction of an activated form of C-RAF. (A, B, D) Expression of indicated genes were assessed by quantitative RT-PCR (A, D) or Western blots (B). Indicated values were averaged from eight PCR reactions. ${ }^{* * *}$ indicates P-values below 0.001. (D, E) Average profile of reads mapping to short-lived (less than $2 \mathrm{~h}$ ) or long-lived (more than $10 \mathrm{~h}$ ) $\mathrm{mRNAs}$ as listed in Tani et al (2012), either in proliferating or in senescent cells as indicated. (F) Ratio of the number of reads mapping to short-lived (less than $2 \mathrm{~h}$ ) over long-lived (more

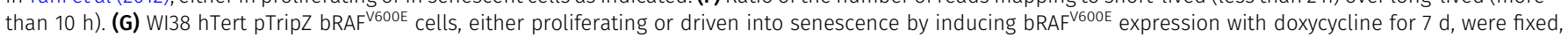


RNA samples in the absence of RNA exosome activity, which may result in less RNA-seq reads per RNA species. The final outcome of these variations was a $40 \%$ increase $(\mathrm{pVal}<0.05)$ in the ratio of the number of reads aligning on short-lived mRNAs over those aligning on long-lived mRNAs (Fig S2E). Henceforth, this ratio will be referred to as the Lo-Hi RNA stability ratio. When applied to the Wi38 data, this approach revealed that the short-lived mRNAs acumulated more in senescent cells than in the proliferating cells, whereas levels of long-lived mRNAs remained unchanged between the two conditions (Fig 2D and E). The resulting Lo-Hi RNA stability ratio was increased by $\sim 40 \%$, similar to that observed in cells depleted for EXOSC3 (Figs 2F and S2E).

Finally, we investigated whether accumulating RNA species also included dsRNAs. To that end, we carried out immunofluorescent staining of Wi38 cells with the 12 anti-dsRNA IgG2a monoclonal antibody (Schonborn et al, 1991). We here used Wi38 cells expressing the activated B-RAF-V600E oncogene under the control of a tetO promoter. In our hands, this system caused a more moderate cytoplasmic contraction in association with RAF-induced senescence, as compared to the cells harboring tamoxifeninducible RAF, and therefore allowed for easier observation of the cytoplasmic compartment. In these experiments, the 12 antibody yielded a clear RNAse-sensitive signal in the cytoplasm of the senescent Wi38 cells, suggestive of an accumulation of dsRNA in that compartment (Fig 2G).

Together, these observations argued in favor of a reduced turnover of unstable RNAs and increased accumulation of dsRNAs in the senescent Wi38 cells, in association with increased activity of innate immune defense mechanisms implicated in defense against dsRNA viruses.

\section{Reduced turnover of unstable RNAs in multiple senescent cells}

To investigate whether the reduced turnover of unstable RNAs observed in the Wi38 cells driven into senescence by oncogenic RAF was an exception or a widespread phenomenon, we examined several additional RNA-seq data sets from senescent cells.

First, we examined another case of oncogene induced senescence involving expression of oncogenic RAS in IMR90 human fibroblasts. In this series, RNA-seq $(n=2)$ was carried out after expression of RAS for 0 , 4, or $10 \mathrm{~d}$ (Lau et al, 2019). Examination of the transcriptome of the cells revealed a progressive reduction in transcripts from several genes encoding subunits of the RNA exosome, including DIS3L, EXOSC2, EXOSC3, EXOSC6, EXOSC8, EXOSC9, and EXOSC10 (Fig 3A). Three subunits were unaffected (DIS3, EXOSC1, and EXOSC7), whereas two non-catalytic subunits were up-regulated (EXOSC4 and EXOSC5). This data set was of insufficient depth to visualize pegeRNAs; we therefore examined (1) the accumulation of unstable compared with stable mRNAs, and (2) the expression of the OAS enzymes. Consistent with a reduced RNA turnover, we found that the Lo-Hi RNA stability ratio increased progressively during the entry of the IMR90 cells into senescence (Fig 3B and C). OAS1 and OAS2 mRNA levels increased accordingly, being, respectively, up-regulated 316fold and 74-fold at $10 \mathrm{~d}$ (Fig 3D). In parallel, expression of DNASE2 and of the DNA receptor TLR9 were increased twofold and sixfold, respectively, at $10 \mathrm{~d}$, whereas the DNAse TREX1 was essentially unaffected. The variations of DNASE2 and TLR9 expression suggest that that these cells may accumulate simultaneously both cytoplasmic DNA and dsRNA.

Next, we examined a data set from human fibroblasts (HCA-2 cells), keratinocytes, and melanocytes driven into senescence by ionizing radiation $(n=6$, with data points at $0,4,10$, and $20 \mathrm{~d}$ of culture) (Hernandez-Segura et al, 2017). In this series, the Lo-Hi RNA stability ratio was gradually increased only in the melanocytes (Fig 3E). These cells were also the only ones to up-regulate the OAS genes, consistent with them having to cope with accumulation of dsRNAs (Fig 3F, 106-fold for OAS1, 182-fold for OAS2, 33-fold for OAS3, and 397-fold for OASL at 20 d). Transcription of the RNA receptors TLR3, IFIH1/MDA5, and DDX58/RIG-I was also increased at all time points in the melanocytes, whereas the HCA2 fibroblasts up-regulated the DNA receptor TLR9 (Fig 3G). Of note, in the melanocytes, it was not possible to unambiguously associate the suspected reduced RNA turnover with reduced transcription of any specific RNA exosome gene. This will be further addressed in the discussion. Finally, this data set also included RNA-seq from quiescent fibroblasts, allowing us to verify that reduced RNA turnover was not triggered by simple growth arrest (Fig 3E-G).

To further probe the robustness of our observations, we finally examined a large data set from Wi38, IMR90, HAEC, and HUVEC cells driven into senescence via different routes ( $n=2$ for each condition), including oncogenic RAS, replicative exhaustion, and DNA damage by treatment with either doxorubicin or $y$ irradiation (Casella et al, 2019). In all experiments on Wi38 cells, senescence led to an increased Lo-Hi RNA stability ratio (Fig S3A). Consistent with this, expression of the OAS genes was up-regulated upon replicative and DNA damage-induced senescence (Fig S3B, top panel). This activation of the OAS genes correlated with increased expression of the TLR3, IFIH1/MDA5, and DDX58/RIG-I sensors of dsRNA (Fig S3B, bottom panel). Interestingly, in the RAS-induced Wi38 senescence, like in the initial RAF-induced Wi38 senescence (Fig $2 \mathrm{C}$ ), transcriptional activation was restricted to OASL and the inflammosome component NLRP3 (Fig S3B, column 1). In all cases, the DNases TREX1 and DNASE2 were affected less than twofold although we noted a three to fourfold activation of the DNA receptor TLR9 in two of the data sets (Dox(2) and $10 \mathrm{~Gy}$ ).

From the same data set, we next analyzed the RNA-seq from three different cells types, HAEC, HUVEC, and IMR90 cells driven into senescence by $y$ irradiation or replicative exhaustion. We observed that the Lo-Hi RNA stability ratio was only moderately increased for the HAEC and HUVEC cells, whereas it was decreased in the IMR90 cells (Fig S3C). The modestly increased Lo-Hi RNA stability ratio in the HAEC and HUVEC cells translated into a small but significant (pVal < 0.05) increase in OAS1, OAS2, TLR3, and IFIH1/MDA5 gene

permeabilized, and analyzed for dsRNA using the mouse monoclonal antibody J2 (red). To visualize the cell nuclei, DNA was stained with DAPI (blue). Where indicated, fixed and permeabilized cells were treated with a cocktail of RNAseA and RNAseH before incubation with the 12 antibody. Scale bar: $5 \mu$ m. Source data are available for this figure. 


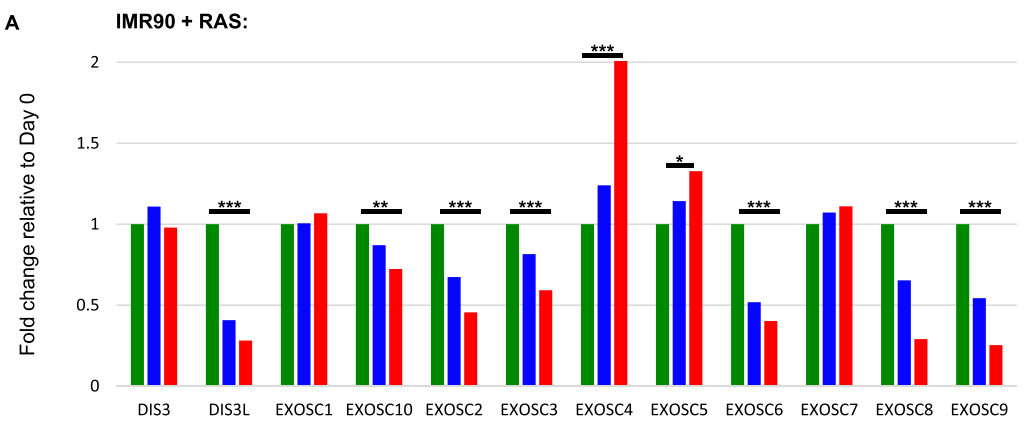

Day 0 Day 4 Day 10
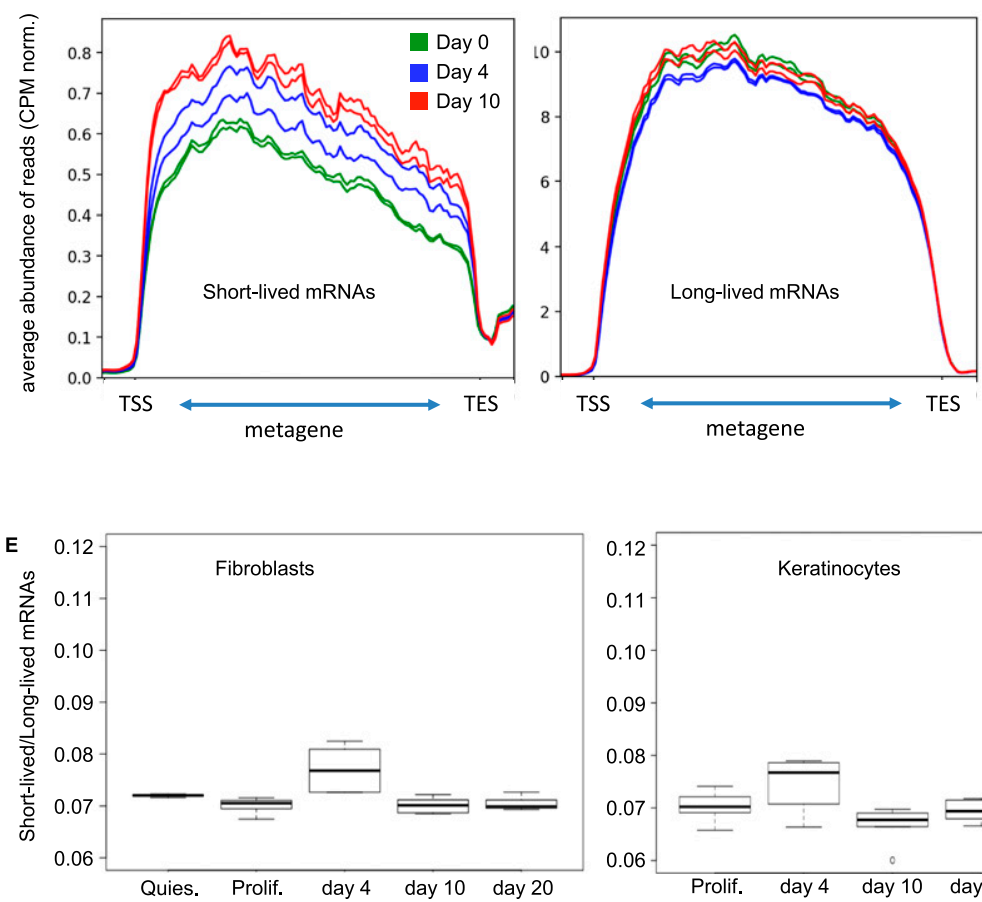

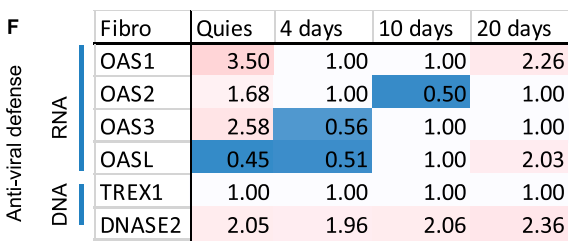

\begin{tabular}{|c|c|c|c|c|c|}
\hline & Fibro & Quies & 4 days & 10 days & 20 days \\
\hline & TLR3 & 1.00 & 0.53 & 0.28 & 0.24 \\
\hline & TLR7 & 1.00 & 1.00 & 1.00 & 1.00 \\
\hline & TLR8 & 1.00 & 1.00 & 1.00 & 1.00 \\
\hline z & IFIH1 & 2.41 & 1.00 & 1.00 & 1.71 \\
\hline & DDX58 & 2.18 & 1.00 & 1.00 & 1.89 \\
\hline & NLRP3 & 0.22 & 1.00 & 0.18 & 1.00 \\
\hline DNA & TLR9 & 3.52 & 3.03 & 5.73 & 7.91 \\
\hline
\end{tabular}

c

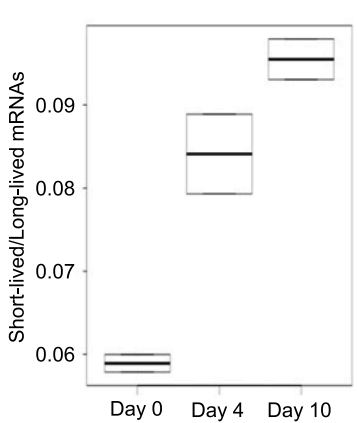

D Fold change relative to Day 0

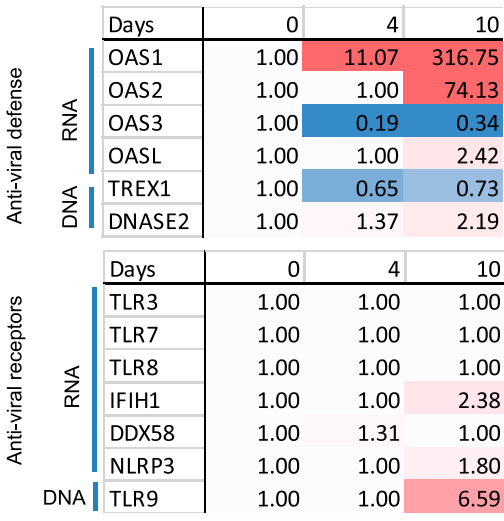
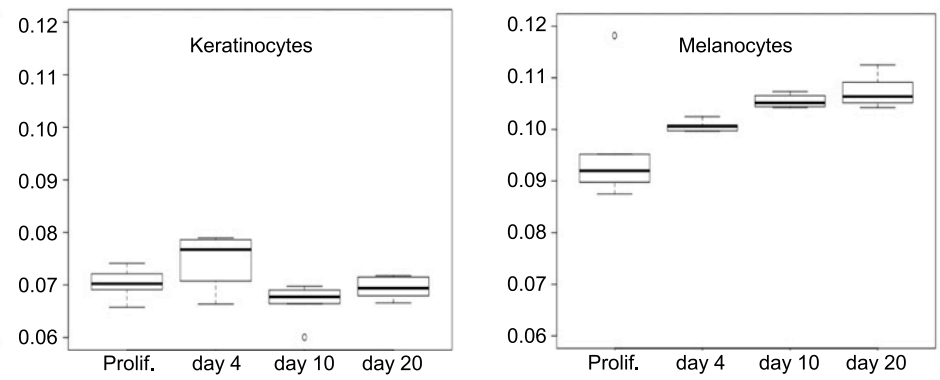

\begin{tabular}{|l|rr|r|}
\hline Keratino & \multicolumn{1}{|c}{ days } & \multicolumn{1}{|c}{ 10 days } & \multicolumn{1}{c}{ 20 days } \\
\hline OAS1 & 1.00 & 1.00 & 0.33 \\
\hline OAS2 & 1.00 & 0.59 & 0.43 \\
\hline OAS3 & 1.00 & 1.00 & 0.26 \\
\hline OASL & 1.00 & 1.00 & 0.31 \\
\hline TREX1 & 0.71 & 1.00 & 0.70 \\
\hline DNASE2 & 0.84 & 0.64 & 0.62 \\
\hline
\end{tabular}

\begin{tabular}{|l|rrr|}
\hline Keratino & 4 days & \multicolumn{1}{|c|}{ 10 days } & \multicolumn{1}{c}{ 20 days } \\
\hline TLR3 & 1.00 & 1.00 & 0.14 \\
\hline TLR7 & 1.00 & 1.00 & 1.00 \\
\hline TLR8 & 1.00 & 1.00 & 1.00 \\
\hline IFIH1 & 1.00 & 1.00 & 0.38 \\
\hline DDX58 & 1.00 & 1.00 & 0.20 \\
\hline NLRP3 & 1.00 & 3.19 & 1.00 \\
\hline TLR9 & 1.00 & 1.00 & 1.00 \\
\hline
\end{tabular}

Fold change relative to proliferating cells

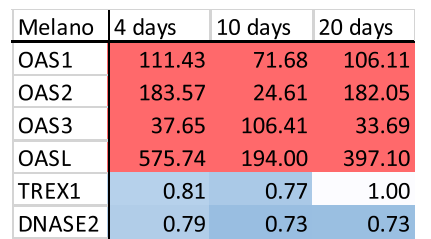

\begin{tabular}{|c|c|c|c|}
\hline Melano & 4 days & 10 days & 20 days \\
\hline TLR3 & 2.02 & 1.64 & 1.98 \\
\hline TLR7 & 1.00 & 1.00 & 1.00 \\
\hline TLR8 & 1.00 & 1.00 & 1.00 \\
\hline IFIH1 & 3.91 & 2.62 & 4.37 \\
\hline DDX58 & 6.97 & 4.91 & 6.77 \\
\hline NLRP3 & 1.00 & 1.00 & 1.00 \\
\hline TLR9 & 1.00 & 1.00 & 2.47 \\
\hline
\end{tabular}

Figure 3. Reduced RNA decay in senescent cells of various origin.

(A, B, C, D) RNA-seq data from IMR90 human fibroblasts induced into senescence via activation of the oncogene Ras (Lau et al, 2019). Samples ( $n=2)$ had been collected at day 0 (growing phase), day 4 (beginning of SASP induction) and day 10 (senescent phase) of Ras induction. (A) Histograms show variations in expression levels of the indicated genes. ${ }^{* * *}$ and ${ }^{* *}$ indicate P-values below 0.001 and 0.01 , respectively. (B) Average profile of reads mapping to short-lived (less than $2 \mathrm{~h}$ ) or long-lived (more than $10 \mathrm{~h}$ ) mRNAs as in Fig 2 at the indicated time points. (C) Lo-Hi RNA stability ratio for the experiment at the indicated time points, calculated as in Fig 2. (D) Fold activation of the indicated genes at the indicated time point. Red gradient indicates up-regulation (max red at 10-fold), blue indicates down-regulation (max blue at 0.5). Variations 
expression, whereas these genes were unaffected or downregulated in the IMR90 cells (Fig S3D).

Altogether, these observations identify a subcategory of senescent cells displaying increased accumulation of the more unstable RNAs and signs of a defense reaction against dsRNA. The variability in transcriptional landscapes observed in the different senescent cells support a model where each cell line is affected by accumulating RNAs to different degrees, possibly as a complement to cytoplasmic accumulation of DNA.

\section{Senescent cells share an RNA signature with cells exposed to oxidative stress}

Accumulation of uaRNAs were previously observed in cells exposed to oxidative stress, a central determinant of senescence (e.g., KLF6 locus in Fig S4A and B and Giannakakis et al [2015], Nilson et al [2017]). This phenomenon was imputed to defective RNA polymerase II transcription termination (Nilson et al, 2017). To investigate possible similarities between oxidative stress and senescence at the level of the RNAs accumulating in the cells, we examined cells exposed to $\mathrm{H}_{2} \mathrm{O}_{2}$ for the presence of pegeRNAs other than uaRNAs. For this study, we chose a data set from $\mathrm{H}_{2} \mathrm{O}_{2}$-treated $\mathrm{BJ}$ or MRC5 cells allowing for detection of rare RNAs (Giannakakis et al, 2015). In these data, like in the RAF-induced Wi38 senescent cells and the EXOSC3-depleted HeLa cells, we observed an accumulation of reads downstream of many histone genes (Figs $4 \mathrm{~A}$ and S4C). We also observed the accumulation of $3^{\prime}$ extensions of $U$ snRNAs (see example in Figs 4B and S4D, and heat map of Fig 4C). Finally, as in the senescent cells, we observed a gradual increase in the Lo-Hi RNA stability ratio, suggestive of a reduced RNA turnover (Figs 4D and S4E).

We next examined the transcriptome of a mouse model involving oxidative stress associated with mitochondrial dysfunction caused by inactivation of the Mof histone acetylase in the heart (Chatterjee et al, 2016). Inactivation of this gene has severe consequences on cardiac tissue that has high-energy consumption, triggering hypertrophic cardiomyopathy and cardiac failure. At the cellular level, cardiomyocytes were reported to show severe mitochondrial degeneration and deregulation of mitochondrial nutrient metabolism and oxidative phosphorylation pathways. Consistent with mitochondrial suffering and ensuing oxidative stress, Mof inactivation reproduced the increased accumulation of uaRNAs observed in the $\mathrm{H}_{2} \mathrm{O}_{2}$-treated human tissueculture cells (see example of the Ryr2 promoter, Fig 4E, and metaplot of 1,200 promoters, Fig 4F). Likewise, Mof inactivation resulted in accumulation of $3^{\prime}$ extensions at $U$ snRNA and histone genes, reproducing another RNA signature of senescence (Fig $4 \mathrm{G}$ and $\mathrm{H}$ ). Consistent with reduced RNA decay, we noted in the Mof KO cells, a significant decrease in the expression of several subunits of the RNA exosome, particularly the catalytic subunit Dis3l, as well as Exosc3, Exosc7, and Exosc9 (Fig 4I). In parallel, and consistent with Mof inactivation causing oxidative stress and DNA damage, we noted a clear increase in the expression of the senescence marker Cdkn1a/p21 (Fig 4J).

Together, these observations suggest that the oxidative stress associated with cells undergoing senescence may be upstream of the accumulation of pegeRNAs observed in these cells.

\section{RNA exosome depletion accelerates the onset of senescence}

To investigate whether inactivation of the RNA exosome had a causative impact on the onset of senescence, Wi38 cells harboring the tetO-B-RAF-V600E construct were transfected with EXOSC3 siRNAs. As previously described, the tetO-B-RAF-V600E system allows modulating the speed at which cells are induced into senescence by reducing the concentration of doxycyclin (Carvalho et al, 2019). Thus, cells received $25 \mathrm{ng} / \mathrm{ml}$ doxycycline for 0, 2, or $3 \mathrm{~d}$ (see schematic Fig 5A and EXOSC3 depletion Fig 5B and C). This short treatment at reduced doxycycline concentrations is not sufficient to induce senescence and did not result in induction of CDKN1A/p21 and CDKN2A/p16 and in only a 10fold induction of CDKN2B/p15 after $3 \mathrm{~d}$ (Fig 5D-F, light-gray bars). In contrast, in cells depleted for EXOSC3, CDKN2A/p16, and CDKN2B/p15 were up-regulated 6 -fold and 25 -fold, respectively, by day 2, suggesting that reduced RNA turnover facilitates the onset of oncogene-induced senescence (Fig 5D-F, dark-gray bars). We note, however, that EXOSC3 depletion alone, in the absence of B-RAF-V600E expression, did not induce senescence markers within the time frame of the experiment.

To gain further evidence for an impact of RNA exosome inactivation on cellular senescence, we examined a data set from mouse embryonic stem cells depleted in Exosc3 for $3 \mathrm{~d}$ (Chiu et al, 2018). As expected and as described in the original article, these cells recapitulated the increased accumulation of uaRNAs (e.g., Fig $5 G)$. They also recapitulated the effect on $U$ snRNA maturation (e.g., Fig $5 \mathrm{H}$ ). Gene Ontology (GO) term analysis of genes up-regulated upon Exosc3 inactivation further revealed a highly significant enrichment in genes associated with the p53 pathway and we noted increased expression of the senescence markers $C d k n 2 b / p 15$, Cdkn2a/p16, and Cdkn1a/p21 (Fig 51 and J and Table S2 listing differentially regulated genes associated with the GO terms). Consistent with this, down-regulated genes were highly enriched in genes associated with cell cycling (Fig 5K). These observations show that loss of RNA exosome activity results in a transcriptional landscape sharing many characteristics with senescent cells.

Examining GO terms for cellular compartments further highlighted a significant enrichment in mitochondrial genes among the genes down-regulated by depletion of Exosc3 (Fig 5L). This was consistent with an earlier report showing mitochondrial dysfunction in patients with pontocerebellar hypotrophy, a syndrome linked to a mutation in the EXOSC3 gene (Schottmann et al, 2017).

Together, these observations indicate that reduced RNA exosome activity induces transcriptional traits characteristic of cellular senescence, possibly favoring the onset of senescence. In addition, the data are suggestive of a bidirectional crosstalk between RNA degradation and oxidative stress for the induction of growth arrest.

with pVal > 0.05 were set to 1. (E, F, G) RNA-seq data from Hernandez-Segura et al (2017), $n=6$ for each cell type and time point. HCA-2 (fibroblasts), keratinocytes, or melanocytes had been exposed to ionizing radiation. RNA had been harvested 4, 10, or $20 \mathrm{~d}$ later. (E) Lo-Hi RNA stability ratio for each experiment calculated as indicated in Fig 2. (D, F, G) Fold activation of the indicated genes represented as in (D). 

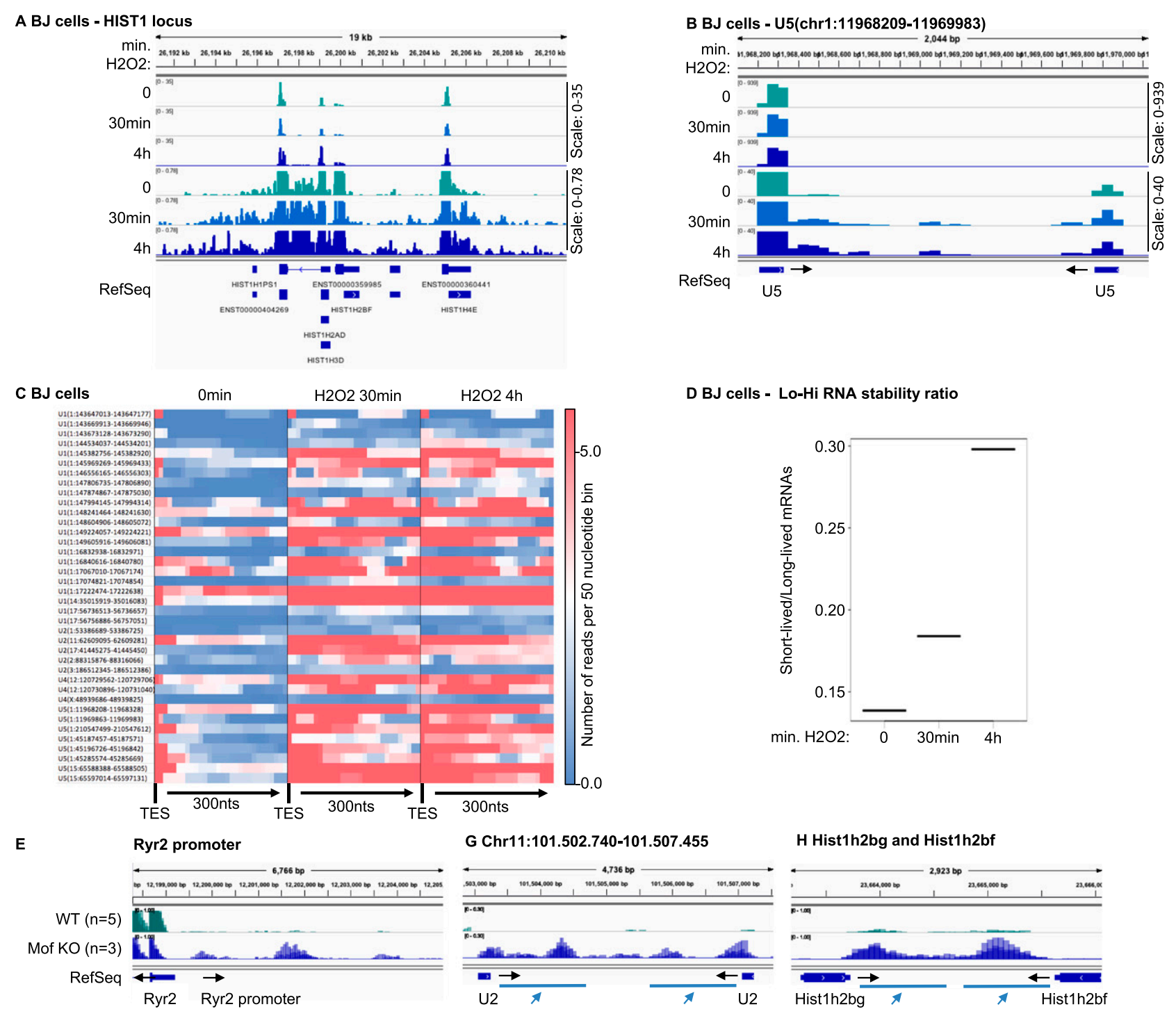

$\mathrm{H} 2 \mathrm{O} 24 \mathrm{~h}$

D BJ cells - Lo-Hi RNA stability ratio

$\mathbf{F}$ meta promoter region in wt and Mof KO mice
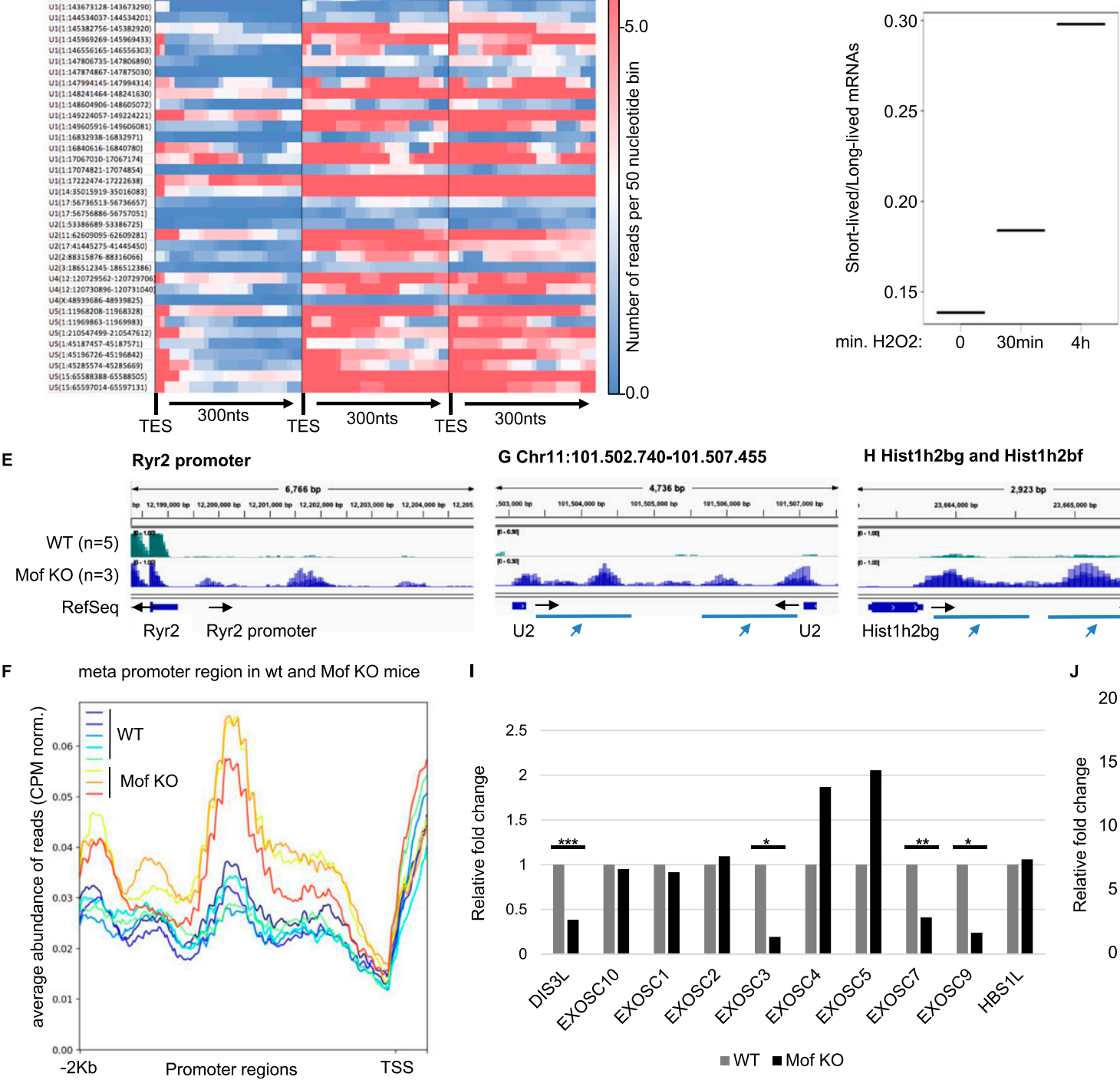

\section{H Hist1h2bg and Hist1h2bf}

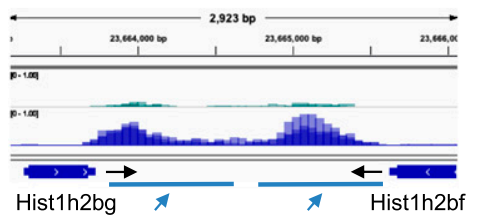

I

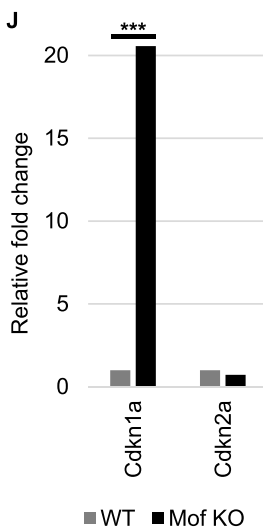

Figure 4. Accumulation of pegeRNAs in cells exposed to oxidative stress.

(A, B, C, D) BJ human fibroblasts exposed to $0.2 \mathrm{mM} \mathrm{H}_{2} \mathrm{O}_{2}$ for the indicated times (one sample per time point) (Giannakakis et al, 2015). Indicated loci were visualized in the Integrative Genomics Viewer genome browser. (C) Heat map illustrating increased accumulation of non-maturated U snRNAs in BJ cells exposed to $\mathrm{H}_{2} \mathrm{O}_{2}$ for the indicated times. Transcription end site indicates the 3' end of the U snRNA gene. (D) Lo-Hi RNA stability ratio for BJ cells at the indicated time points, calculated as indicated in Fig 2. (E, F, G, H, I, J) Mouse cardiomyocytes either WT ( $n=4$ ) or inactivated for the Mof histone acetylase ( $n=3$ ) were analyzed by RNA-seq (Chatterjee et al, 2016). (E, G, H) Data were aligned on mouse genome mm9 and indicated loci were examined using the Integrative Genomics Viewer genome browser. (F) Average profile of read distribution along the promoters of 1,200 genes with similar expression levels in either WT or Mof KO cardiomyocytes. (I, J) Differential gene expression was estimated with DESeq2. Histograms show variations of the indicated genes. ${ }^{\star * *}$, ${ }^{\star *}$, and * indicate $P$-values below $0.001,0.01$, and 0.05 respectively. 


\section{Serendipitous transcription of DNA repeats in pegeRNAs}

We finally investigated how pegeRNAs may trigger cytoplasmic antiviral mechanisms. In this context, we noted that an earlier study had reported an accumulation in senescent cells of RNAs originating from the SINE family of retrotransposons (Wang et al, 2014). In parallel, another study showed that SINE-containing RNAs can trigger an interferon response and stimulate secretion of cytokines (Hung et al, 2015). Together, these observations prompted us to investigate whether pegeRNAs may be enriched in SINE or other repeated sequences prone to yield dsRNAs detectable by the interferon pathway.

To investigate this possibility, the average distribution of SINES and LINEs in regions producing pegeRNAs was examined by plotting the profile of these repetitive elements over all proteincoding genes (annotated NM in the RefSeq database). This revealed that the density in SINES and LINES was low at transcription start site (TSS) and transcription end site, but rapidly increased when moving away from these sites (Fig 6A). Likewise, density in SINEs and LINES was low over U snRNA gene bodies, but then returned to average levels within a few hundred nucleotides from the gene boundaries (Fig 6B). Thus, SINE and LINE repeats have a distribution compatible with them being absent from short promoter RNAs and short 5' extensions, but present within the longer pegeRNAs.

To document this, we plotted the average distribution of reads at promoters in Wi38 cells driven into senescence by oncogenic RAF (Fig 6C), or in BJ cells exposed to $\mathrm{H}_{2} \mathrm{O}_{2}$ (Fig 6D). In these plots, we scaled the region from the TSS to the first SINE to a fixed $1 \mathrm{~Kb}$. This allowed us to visualize transcription before and after the first SINE upstream of the TSS. In both data sets, promoter transcription rarely reached beyond the first SINE in proliferating cells (Fig 6C and D, dark-blue lines), whereas this apparent first-SINE limit was readily crossed in the senescent cells and even more so in the cells exposed to oxidative stress (Fig 6C and D, light-colored lines).

These observations define pegeRNAs as a source of transcripts containing transposon sequences, and we speculate that these transposons could be a source of dsRNA detected by the antiviral defense mechanisms.

\section{Discussion}

Senescent cells have many characteristic phenotypes, including growth arrest, modified chromatin structure, and secretion of pro-inflammatory molecules. Here, we document an additional characteristic, namely, the accumulation of RNA species transcribed from regions located upstream and downstream of genes. These RNA species that we refer to as perigenic RNAs (pegeRNAs), include uaRNAs and 3' extensions of genes. These RNAs were found to accumulate in multiple cell lines, driven into senescence via multiple routes, including forced expression of oncogenes, DNA damage, and replicative exhaustion.

$3^{\prime}$ extensions of genes are removed from the main transcript during $U$ snRNA maturation. The cleaved product is then degraded by the RNA exosome (Allmang et al, 1999). Accumulation of reads downstream of genes in HeLa cells depleted in EXOSC3, as well as observations in yeast (Lemay et al, 2014; Villa et al, 2020), suggest that the RNA exosome also participates directly or indirectly in transcription termination of mRNAs. Finally, the RNA exosome clears the cells of uaRNAs (Ogami et al, 2018). Thus, the different RNA species composing pegeRNAs are all likely targets of this RNA degradation machinery. Accordingly, we observed reduced expression of RNA exosome subunits in two of the senescent cell lines, namely, the Wi38 and the IMR90 cells driven into senescence by oncogenic RAF and RAS, respectively. In both cases, expression of the DIS3L gene coding for the catalytic subunit of the cytoplasmic RNA exosome was affected. Expression of this subunit was also down-regulated in the cells exposed to chronic oxidative stress in the mouse model inactivated for Mof. In the IMR90 and in the mouse cells, expression of the regulatory subunits EXOSC3 and EXOSC9 were also down-regulated. In contrast, the non-catalytic subunit EXOSC4 was up-regulated in all three cellular models, for reasons which are as yet unclear.

In the other senescent cells examined, expression of DIS3L was down-regulated less than twofold. We speculate that in these cells, the reduced RNA decay may also involve post-translational mechanisms. The existence of such mechanisms is suggested by the very rapid accumulation of pegeRNAs (30 min) observed in cells exposed to $\mathrm{H}_{2} \mathrm{O}_{2}$ (this article and original analysis by Giannakakis et al [2015]). Similarly, the half-life of unstable ncRNAs was previously shown to be drastically increased in cells exposed to oxidative stress (Tani et al, 2019).

To estimate RNA decay efficiency, we took advantage of the differential sensitivity of stable and unstable mRNAs to changes in the activity of RNA degradation machineries. This approach was verified in cells with reduced RNA exosome activity caused by depletion of EXOSC3. Calculating the ratio between reads mapping to unstable RNAs and stable RNAs (Lo-Hi RNA stability ratio) allowed us to address changes in RNA decay efficiency even in RNA-seq data sets of insufficient depth for the detection of the rare pegeRNAs or carried out on poly(A) selected libraries. This LoHi RNA stability ratio does not provide any direct information on the activity of the RNA exosome, but may be of general interest as a first approach to the analysis of RNA decay in, for example, cancer cells.

An increased Lo-Hi RNA stability ratio was associated with increased expression of OAS genes in essentially all the senescent cells we examined. We speculate that this is a manifestation of cells facing accumulation of dsRNAs in the cytoplasm. Indeed, we observed dsRNAs accumulating in the cytoplasm of senescent Wi38 cells. In addition, an earlier study has shown that pegeRNAs are extensively detected in the polysomes of cells exposed to $\mathrm{H}_{2} \mathrm{O}_{2}$ (Giannakakis et al, 2015). Possibly, the high stability of these RNAs in cells exposed to stress may increase the probability of their export from the nucleus to the cytoplasm.

Examination of the transcriptomes also provided clues as to the receptors possibly involved in the detection of the stabilized RNAs. Indeed, we noted that up-regulation of the interferon-inducible OAS genes was frequently associated with increased expression of either TLR3, IFIH1/MDA5, and DDX58/RIG-I genes, which encode RNA sensors upstream of the interferon pathway, or of NLRP3, the inflammosome sensor protein. Although the regulation of these RNA sensors is poorly documented, their increased transcription may reflect an increased need for RNA detection in the lengthy process of entering senescence, or it may represent an auto-amplification mechanism. 
A

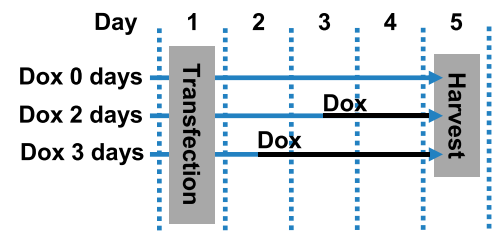

B

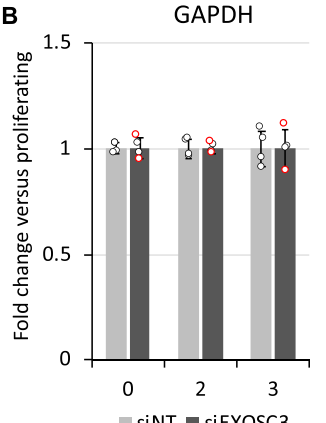

siNT $\square$ SiEXOSC3

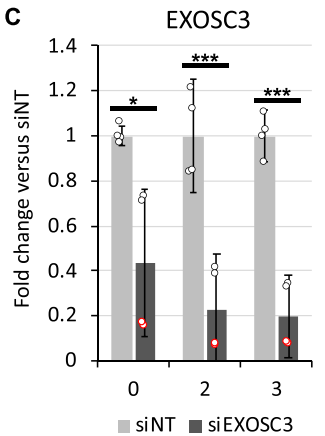

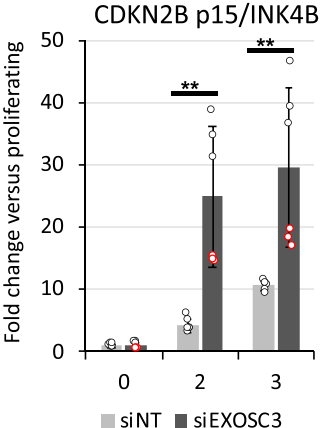

E

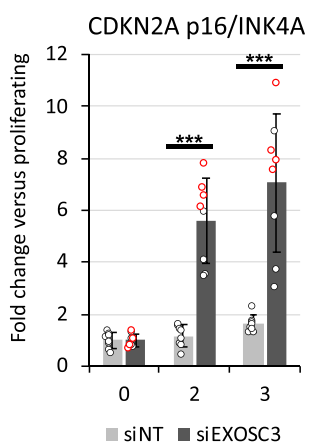

F

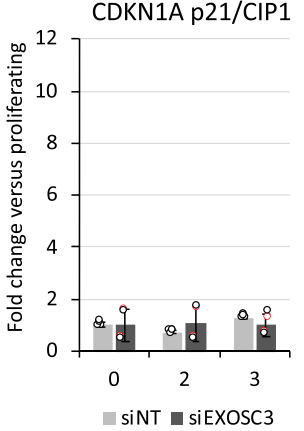

Actb
H

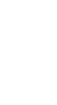

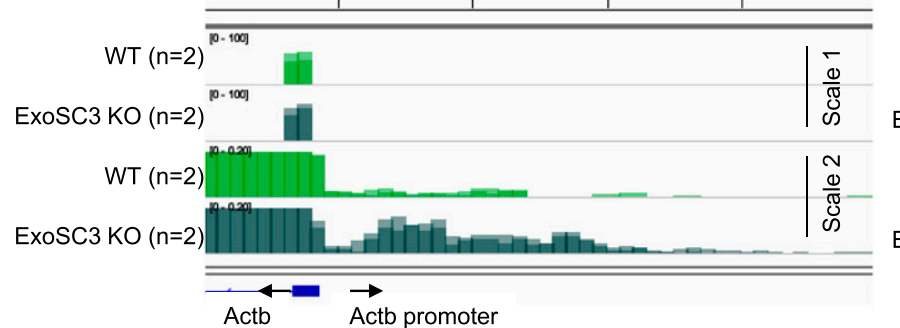

I

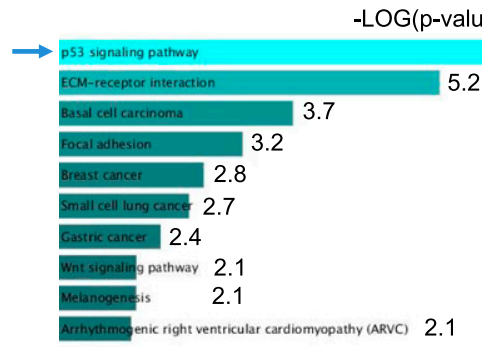

$\mathbf{K}$

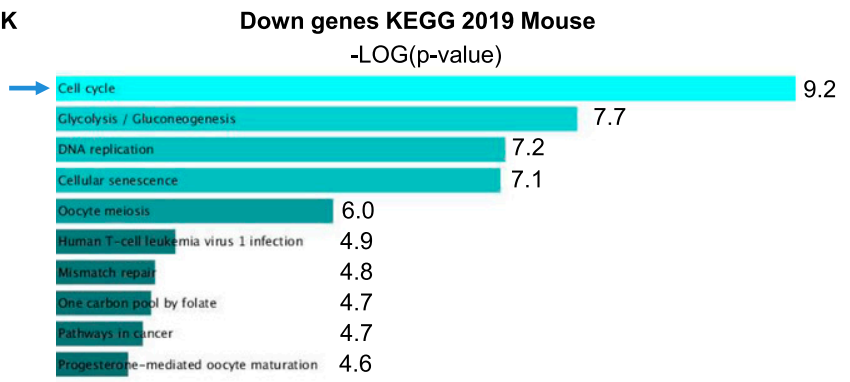

$(n=2)$

chr11:101,502,811-101,507,564

$\longleftarrow$ 4,721 bp

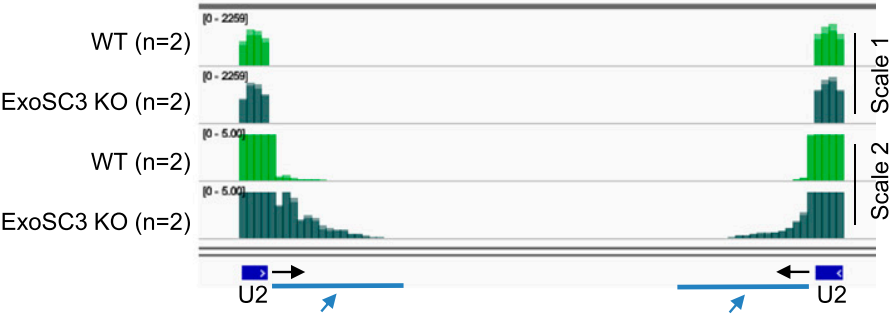

J

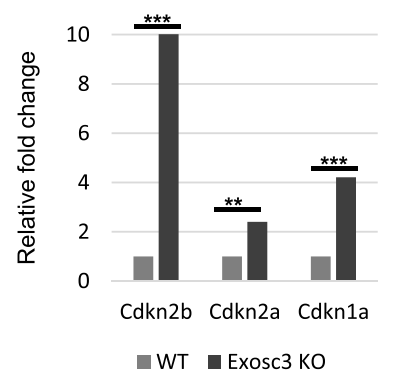

L Down genes Go Cellular Component

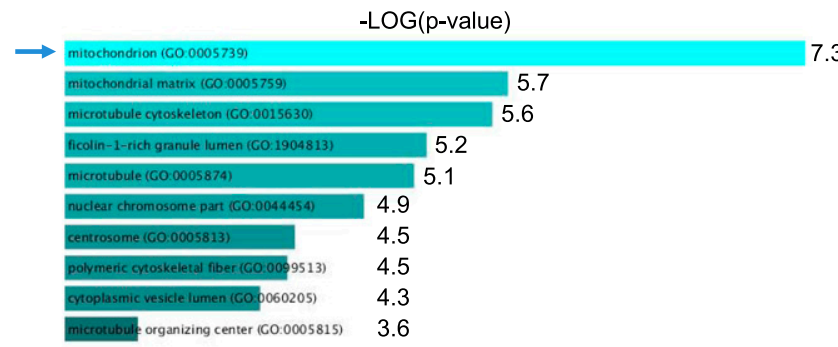

Figure 5. Inactivation of the RNA exosome induce a senescent-like phenotype and deregulates mitochondrial genes.

(A) Schematic: WI38 hTert pTripZ bRAF ${ }^{\mathrm{V} 600 \mathrm{E}}$ cell were transfected with either non-targetted siRNAs (siNT) or two different EXOSC3 siRNAs (red and white dots in histograms), then treated with $25 \mathrm{ng} / \mathrm{ml}$ of doxycycline at the time indicated in the schematic. All samples were harvested and the same time, and total RNA was extracted. (B, C, D, E, F), Expression of indicated genes were assessed by quantitative RT-PCR. Indicated values were averaged from eight PCR reactions. ${ }^{* *}$, and ${ }^{* *}$ indicate $P$-values below 0.001 , and 0.01 respectively. (G, $\left.\mathbf{H}, \mathbf{I}, \mathbf{J}, \mathbf{K}, \mathbf{L}\right)$ RNAseq data $(n=2)$ from mouse ES cells inactivated for Exosc3 and harboring an inducible Exosc3 expression construct human (Chiu et al, 2018). Exosc3 expression is initially induced (WT) then the induced is removed from the medium and cells are cultured for $3 \mathrm{~d}$ (Exosc3 KO). (G, $\mathbf{H}$ ) Indicated loci were visualized with Integrative Genomics Viewer. Black arrows indicate the orientation of the gene. Blue arrows indicate regions of interest. (I, K, L) GO term analysis of genes differentially expressed upon Exosc3 KO was carried out with Enrichr. (J) Differential gene expression was estimated with DESeq2. Histograms show variations of the indicated genes. ${ }^{\star *}$, and ${ }^{* *}$ indicate P-values below 0.001 , and 0.01 respectively. 


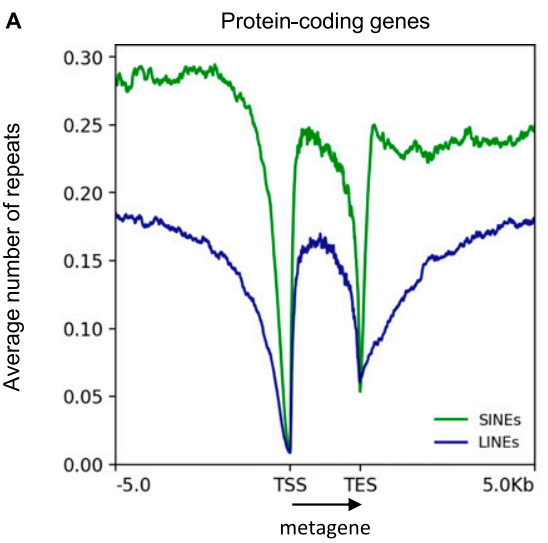

C

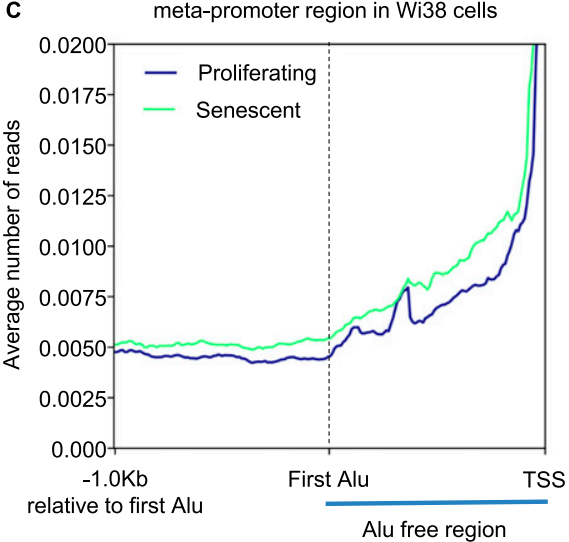

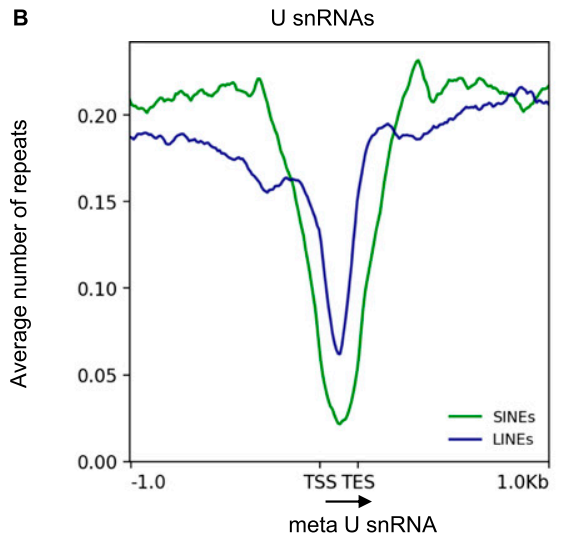

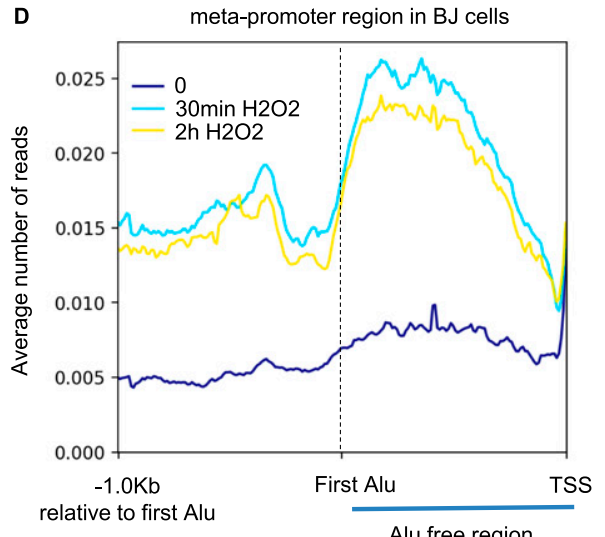

E Model

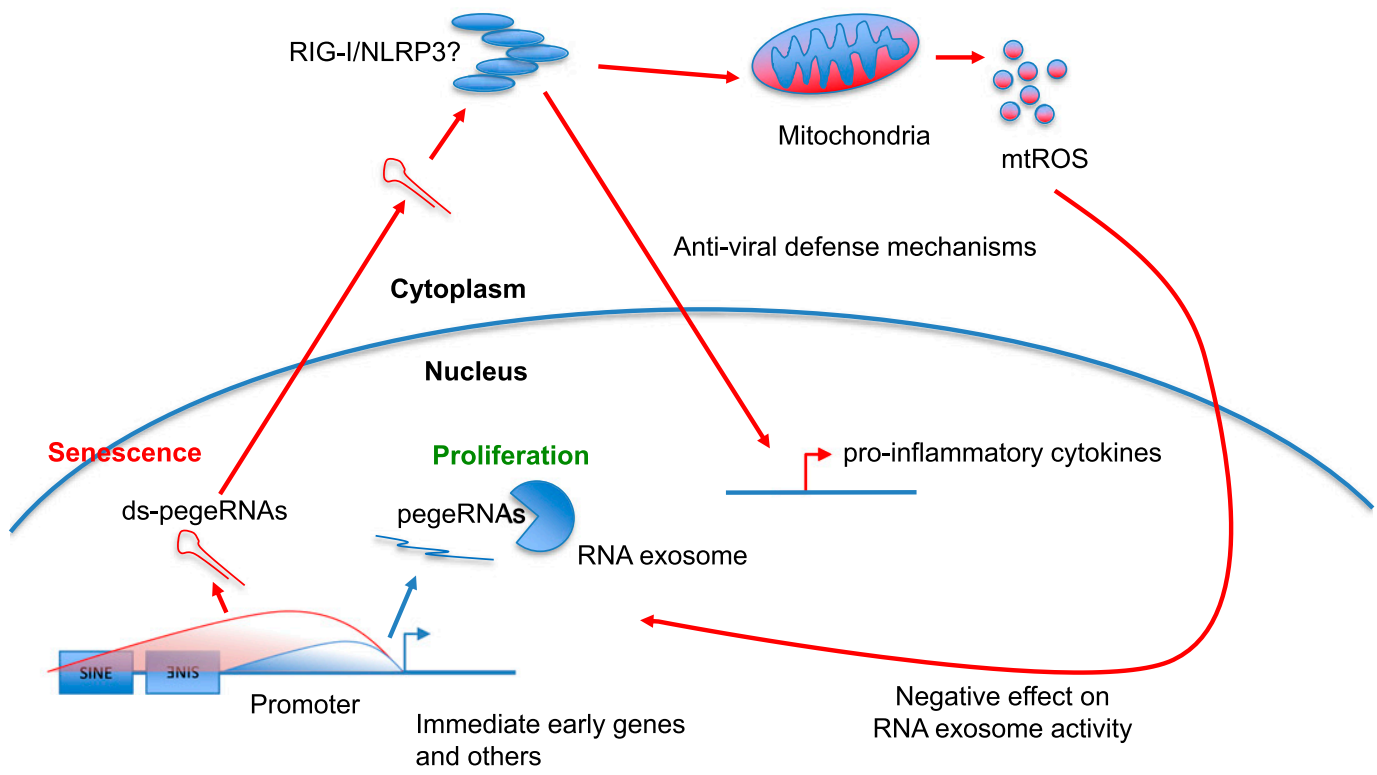

Figure 6. pegeRNAs from senescent cells reach into SINE- and LINE-containing regions.

(A, B) Average number of either SINEs (green profiles) or LINEs (blue profiles) per 50 nucleotide bin in the neighborhood of either protein-coding genes (A) or snRNAs (B). TSS, transcription start site; TES, transcription end site. Gene bodies are all scaled to $2 \mathrm{~Kb}$. U snRNA bodies are scaled to 200 nucleotides. Location of SINES and LINEs was obtained from the Hg19 version of RepeatMasker. (C, D) At the 5,260 promoters not overlapping with coding regions of any gene described in Fig 1 , the region from the TSS to the first Alu sequence was scaled to $1 \mathrm{~Kb}$ (indicated as Alu-free region). The profile then continues $1 \mathrm{~Kb}$ after the start of this first Alu. The average number of reads per 50 nucleotide bin was then calculated within and upstream of this region. (C, D) This was carried out for the RNA-seq data from (C) WI38 hTERT RAF1-ER human fibroblasts 
NLRP3 was reported to be activated by Alu RNAs (Tarallo et al, 2012). RNAs encoded by these retrotransposons have previously been shown to stimulate secretion of cytokines (Hung et al, 2015). This emphasizes the possible role of repeated motifs originating from retrotransposons in the activity of pegeRNAs. In senescent cells and cells exposed to oxidative stress, pegeRNAs will harbor sequences from transposable elements as the transcripts extend beyond the repeat-free promoter and termination regions. In turn, these sequences will potentially be subject to direct detection by, for example, Ro60/TROVE2 that binds inverted Alu sequences (Hung et al, 2015), or will fold into dsRNAs if the transposons form inverted repeats.

Among the senescent cells we examined, we also identified four cases for which the Lo-Hi RNA stability ratio was either unchanged or decreased, namely the fibroblasts IMR90 and HCA-2, and the keratinocytes driven into senescence by $\gamma$ irradiation, and the IMR90 fibroblasts subject to replicative senescence. In these cells, the DNA/CGAS/STING1 pathway may be dominant in triggering the inflammatory phenotype. This leads us to propose that DNA and pegeRNAs may be two alternative and possibly complementary drivers of senescence.

An active role for pegeRNAs in the onset of senescence is suggested by the accelerated production of senescence markers that we observed in Wi38 cells depleted in EXOSC3 with siRNAs and then challenged with activated RAF. In this experiment, we also noted that EXOSC3-depletion alone was not sufficient to trigger the senescence markers. This may, however, be due to the transient nature of siRNA depletion. Indeed, mining of publicly available data showed that inactivation of the Exosc3 gene in mouse ES cells triggered several senescence associated phenotypes, including activation of Cdkn1a/p21, Cdkn2a/p16, and Cdkn2b/ p15, activation of the p53 pathway, and reduced expression of multiple markers of cell cycle progression, all in favor of a triggering effect of pegeRNA.

Inactivation of Exosc3 in the mouse ES cells resulted also in reduced expression of mitochondrial genes. Interestingly, medical data recapitulate this observation. Indeed, patients suffering from pontocerebellar hypoplasia, a disease affecting the development of the brain and due to a mutation in the EXOSC3 gene were also described as showing signs of mitochondrial dysfunction (Schottmann et al, 2017). In parallel, patients with mutations in EXOSC2 are subject to premature aging (Di Donato et al, 2016). TLR3, IFIH1/MDA5, DDX58/RIG-I, and NLRP3 are all connected with mitochondria (Djafarzadeh et al, 2011; Zhou et al, 2011), whereas mitochondrial dysfunction is a source of oxidative stress, which in turn is a trigger of pegeRNA accumulation. We suggest that together, these elements compose a possible feedback loop in which accumulation of pegeRNAs triggers antiviral defense mechanisms causing production of mitochondrial reactive oxygen species that in turn nurtures both the inflammatory response and the reduced RNA turnover. Once initiated, such a process could be an irreversible driver of cellular senescence (Model Fig 6E).

\section{Materials and Methods}

\section{Tissue culture}

WI38 hTERT RAF-ER cells, which are immortalized by hTERT expression and contain an inducible RAF1 oncogene fused to the estrogen receptor (ER), were maintained in MEM supplemented with glutamine, nonessential amino acids, sodium pyruvate, penicillin-streptomycin, and $10 \%$ fetal bovine serum in normoxic culture conditions $\left(5 \% \mathrm{O}_{2}\right.$ ) (Jeanblanc et al, 2012). For the induction of oncogene-induced senescence, the cells were treated with $20 \mathrm{nM}$ 4-HT (H7904; Sigma-Aldrich) for 3 d. WI38 hTert pTripZ bRAFV600E cells that express bRAF ${ }^{\mathrm{V} 600 \mathrm{E}}$ under the control of a tetO promoter were cultivated likewise. RAF expression was induced by either 25 $\mathrm{ng} / \mathrm{ml}$ (siRNA experiment) or $200 \mathrm{ng} / \mathrm{ml}$ (immunofluorescence) of doxycycline (D3447; Sigma-Aldrich).

\section{Immunofluorescent staining}

Proliferating WI38 hTert pTripZ bRAFV600E cells, and the same cells induced into senescence by incubation with $200 \mathrm{ng} / \mathrm{ml}$ of doxycycline for $7 \mathrm{~d}$, were washed with PBS and then fixed with $1.6 \%$ formaldehyde for $15 \mathrm{~min}$. Cells were grown on collagen-coated cover slips in 24-well plates. Cells were washed twice with PBS and permeabilized by incubation with PBS $0.2 \%$ Triton X-100 for 5 min. One set of senescent cells was then treated with a mix of $40 \mathrm{U} /$ $\mathrm{ml} \mathrm{RNaseA}+0.25 \mathrm{mg} / \mathrm{ml}$ RNaseH in PBS $+50 \mathrm{mM} \mathrm{MgCl} 2$ for $3 \mathrm{~h}$ at $37^{\circ} \mathrm{C}$. All samples were then washed three times with PBS $+0.1 \%$ Tween-20 $+0.05 \%$ Triton X-100 and then incubated with a blocking buffer consisting of PBS $+5 \%$ BSA $+0.1 \%$ Tween-20 for $1 \mathrm{~h}$ at RT. Cells were then incubated with a 1/200 solution of mouse monoclonal 12 anti-dsRNA antibody (Scicons) for $1 \mathrm{~h} 30 \mathrm{~min}$ at RT. in blocking buffer. Cells were washed three times with PBS $+0.1 \%$ Tween-20 + 0.05\% Triton X-100 and then incubated with a 1/500 dilution of Alexa-594 antimouse secondary antibody in blocking buffer for $1 \mathrm{~h}$ at RT. Cells were washed three times with PBS $+0.1 \%$ Tween-20 and then stained for $5 \mathrm{~min}$ with $0.25 \mu \mathrm{g} / \mathrm{ml}$ DAPI in PBS. Cells were washed once with PBS and then mounted in an antifading mounting solution on microscope slides. Images were acquired with a Leica SP8 confocal microscope with a 63x objective. The images were all acquired with identical settings.

either proliferating or driven into senescence (Lazorthes et al, 2015) or (D) BJ cells exposed to $\mathrm{H}_{2} \mathrm{O}_{2}$ for the indicated times (Giannakakis et al, 2015). (E) Hypothetical model: an initial source of oxidative stress increases elongation and reduces degradation of pegeRNAs that will eventually contain sequences encoded by repeats originating from retrotransposons. Because of their abundance, a fraction of the pegeRNAs reaches the cytoplasm (Giannakakis et al, 2015). In the cytoplasm, dsRNAs generated by inverted repeats are detected by antiviral defense mechanisms. Activation of these RNA receptors results in mitochondrial dysfunction (Djafarzadeh et al, 2011). This leads to production of mitochondrial reactive oxygen species (mtROS) that hampers the RNA exosome activity and feeds the inflammatory phenotype of senescent cells. 


\section{Western blotting}

Cells were lysed with lysis buffer $(50 \mathrm{mM}$ Tris- $\mathrm{HCl}, \mathrm{pH}$ 7.5, $150 \mathrm{mM}$ $\mathrm{NaCl}, 1 \%$ Triton X-100, 0.1\% SDS, 1 mM EDTA, and protease/phosphatase inhibitor mixture [Roche]) and sonicated (Bioruptor; Diagenode). The protein concentration was determined by Bradford assay, and $20 \mu \mathrm{g}$ of protein were boiled in the presence of NuPAGE lithium dodecyl sulfate sample buffer (NP0007; Invitrogen) and NuPAGE Sample Reducing Agent (NU0004; Invitrogen) at $95^{\circ} \mathrm{C}$ for $5 \mathrm{~min}$, resolved by SDS-PAGE (4-12\% Criterion XT Bis-Tris Protein Gel; Bio-Rad), and transferred to nitrocellulose membrane (Bio-Rad). Staining with ATX Ponceau S Red (09189; Sigma-Aldrich) was used as a further marker of protein content. The membrane was then blocked with 5\% non-fat milk in PBS-0.1\% Tween-20 (P1379; Sigma-Aldrich) for 1 $h$ at RT and probed with specific primary antibodies ( $\alpha$-p21 [1:500, 556430; BD Biosciences], DIS3L [1:500, ab89042], and actin [1:1,000, A2103; Sigma-Aldrich]) overnight at $4^{\circ} \mathrm{C}$. After three washes in PBS containing $0.1 \%$ Tween 20 , the membrane was incubated with antirabbit or antimouse IgG HRP secondary antibodies for $1 \mathrm{~h}$ at RT and revealed by chemiluminescence, respectively. Detection was performed using Chemidoc MP imaging system (Bio-Rad). Experiments were performed in triplicate, and a representative Western blot was shown. Quantification of Western blot bands was performed using the ImageJ software.

\section{Mapping}

For the mapping, SHRiMP was used (v2.2.3) (David et al, 2011) for the data set GSE55172 (color space reads) (parameters: -o 1 -maxalignments 10 -strata), whereas for the others, mapping was performed with STAR (v2.6.0b) (Dobin et al, 2013) (parameters: -outFilterMismatchNmax 1 -outSAMmultNmax 1 -outMultimapperOrder Random -outFilterMultimapNmax 30). Mapping was performed against the reference human genome (hg19 homo sapiens primary assembly from Ensembl) for the European Bioinformatics Institute data set, GSE55172, GSE81662, GSE85085, GSE108278, GSE130727, and against the reference mouse genome ( $\mathrm{mm} 9$ mus musculus primary assembly from Ensembl) for the GSE77784 and GSE100535. The SAM files were then converted to the BAM format and sorted by coordinate with SAMtools (v1.7) (Li et al, 2009).

\section{Data observations}

Bigwigs files were generated with bamcoverage (parameter: -normalizeUsing CPM) from deepTools (v3.1.3) (Ramirez et al, 2016). All observations were done using the Integrative Genomics Viewer software (Robinson et al, 2011).

\section{Differential gene expression}

The package Subread (v1.28.1) (Liao et al, 2014) for R (v3.4.3) was used to count the uniquely mapped reads based on a gtf annotation file for hg19 or mm9 from Ensembl. Then the package DESeq2 (v1.18.1) (Love et al, 2014) was used to make the differential gene expression analysis and principal component analysis. P-values from the differential gene expression test were adjusted for multiple testing according to the Benjamini and Hochberg procedure. Only genes with an adjusted $P$-value lower than 0.05 were considered differentially expressed. GO term analysis were performed on these differentially expressed genes with Enrichr (Kuleshov et al, 2016).

\section{Heat maps and profiles}

Reads inside upstream gene regions and downstream $U$ snRNA regions were quantified using featureCounts (v1.6.1) from the Subread suite (Liao et al, 2014).

Then, from these counts, matrices were generated with the tool computeMatrix reference-point (parameter: -referencePoint TSS for the observations of the regions upstream of the genes or -referencePoint transcription end site for the observations of the downstream regions of $U$ snRNA). Profiles were obtained using plotProfile (parameter: -perGroup) and heat maps using plotHeatmap (default paramaters) from the deepTools suite (Ramirez et al, 2016).

\section{Calculation of the Lo-Hi RNA stability index}

The package Subread (v1.28.1) (Liao et al, 2014) for R (v3.4.3) was used to count the uniquely mapped reads based on a list of either highly unstable (t1/2 < 2 h) or highly stable (t1/2 > $10 \mathrm{~h}$ ) mRNAs from Tani et al (2012). For each feature, the count was normalized using the Transcript per Million method. Then, for both conditions, the sum of every Transcript per Million was calculated and the highly unstable mRNAs was divided by the highly stable mRNAs, giving us a value for this Lo-Hi RNA stability index. Bed files of the highly unstable and highly stable mRNAs are available as supplemental data.

\section{Quantitative RT PCR and siRNA knock-down}

Cells were transfected in six-well plates with Lipofectamine RNAiMax (Invitrogen, Thermo Fisher Scientific) and 10 nM siRNA duplexes (Sigma-Aldrich) following the manufacturer's instructions. Total RNA was prepared by guanidinium thiocyanate-phenol-chloroform extraction (Chomczynski \& Sacchi, 2006). After DNAse treatment 20 min at $37^{\circ} \mathrm{C}$, reverse transcription was carried out with SuperScript III (Invitrogen) and random hexanucleotides for $1 \mathrm{~h}$ at $42^{\circ} \mathrm{C}$ on $0.5 \mu \mathrm{g}$ RNA, quantified with a nanodrop (Thermo Fisher Scientific). Realtime qPCR was carried out on a Stratagene M×3005p with Brilliant III SYBR Green kits (Stratagene) according to the manufacturer's instructions.

\section{SiRNAs}

siEXOSC3 duplex 1: CACGCACAGUACUAGGUCA (Preker et al, 2008), siEXOSC3 duplex 2: GACGUCAGAUCAAAGAAAA, siNT: universal negative control \#1 siRNA duplex (Sigma-Aldrich, ref SIC001).

\section{Primer sequences}

CDKN1A-F CACTCAGAGGAGGCGCCATGTCA Amplicon Length: 213 bp CDKN1A-R CCCAGGCGAAGTCACCCTCCA

CDKN2A-F CACCGAATAGTTACGGTCGG Amplicon Length: 129 bp 
CDKN2A-R GCACGGGTCGGGTGAGAGTG

CDKN2B-F GGACTAGTGGAGAAGGTGCG Amplicon Length: 106 bp CDKN2B-R GGGCGCTGCCCATCATCATG

OASL-F ATGTTGGACGAAGGCTTCACCACT Amplicon Length:193 bp

OASL-R ATCTGTACCCTTCTGCCACGTTGA

NLRP3-F CGATCAACAGGAGAGACCTTTAT Amplicon Length: 108 bp

NLRP3-R TGCTGTCTTCCTGGCATATC

DIS3L-F ACTCCGGGAATGTGCTAAAG Amplicon Length: 117 bp

DIS3L-R AGTAGCCTGTTCACAATGGG

EXOSC3-F CTCTCAGCAGAAGCGGTATG Amplicon Length: 103 bp

EXOSC3-R CTCACTCCCTCCAACATCAAC

GAPDH-F ATGGGGAAGGTGAAGGTCG Amplicon Length: 108 bp

GAPDH-R GGGGTCATTGATGGCAACAATA

\section{Data Availability}

RNA-seq raw fastq files from GSE55172, GSE77784, GSE81662, GSE85085, GSE100535, GSE108278, and GSE130727 were downloaded from Gene Expression Omnibus on the National Center for Biotechnology Information database. The fastq files from E-MTAB-5403 were downloaded from ArrayExpress on the European Bioinformatics Institute database.

\section{Supplementary Information}

Supplementary Information is available at https://doi.org/10.26508/lsa. 202000809.

\section{Acknowledgements}

We thank Régis Courbeyrette for technical assistance and M Ricchetti for critical reading of the manuscript. C Muchardt was supported by grants from Institut Pasteur (PTR No 24-17), LABEX REVIVE, and Agence Nationale de la Recherche (ANR 15-CE14-0003). C Mann was supported by ANR 17008-02.

\section{Author Contributions}

N Mullani: investigation.

Y Porozhan: investigation.

A Mangelinck: investigation and methodology.

C Rachez: validation, investigation, methodology, and writingreview and editing.

M Costallat: formal analysis.

E Batsché: investigation and methodology.

M Goodhardt: funding acquisition, methodology, and writingreview and editing.

G Cenci: funding acquisition, methodology, and writing-review and editing.

C Mann: conceptualization, formal analysis, funding acquisition, investigation, methodology, and writing-review and editing. C Muchardt: conceptualization, data curation, formal analysis, supervision, funding acquisition, investigation, methodology, project administration, and writing-original draft, review, and editing.

\section{Conflict of Interest Statement}

The authors declare that they have no conflict of interest.

\section{References}

Allmang C, Kufel J, Chanfreau G, Mitchell P, Petfalski E, Tollervey D (1999) Functions of the exosome in rRNA, snoRNA and snRNA synthesis. EMBO J 18: 5399-5410. doi:10.1093/emboj/18.19.5399

Ben-Porath I, Weinberg RA (2005) The signals and pathways activating cellular senescence. Int J Biochem Cell Biol 37: 961-976. doi:10.1016/ j.biocel.2004.10.013

Cao K, Blair CD, Faddah DA, Kieckhaefer JE, Olive M, Erdos MR, Nabel EG, Collins FS (2011) Progerin and telomere dysfunction collaborate to trigger cellular senescence in normal human fibroblasts. I Clin Invest 121: 2833-2844. doi:10.1172/jci43578

Carvalho C, L'Hote V, Courbeyrette R, Kratassiouk G, Pinna G, Cintrat JC, Denby-Wilkes C, Derbois C, Olaso R, Deleuze JF, et al (2019) Glucocorticoids delay RAF-induced senescence promoted by EGR1. $J$ Cell Sci 132: jcs230748. doi:10.1242/jcs.230748

Casella G, Munk R, Kim KM, Piao Y, De S, Abdelmohsen K, Gorospe M (2019) Transcriptome signature of cellular senescence. Nucleic Acids Res 47 7294-7305. doi:10.1093/nar/gkz555

Cecco M, Ito T, Petrashen AP, Elias AE, Skvir NJ, Criscione SW, Caligiana A, Brocculi G, Adney EM, Boeke JD, et al (2019) L1 drives IFN in senescent cells and promotes age-associated inflammation. Nature 566: 73-78. doi:10.1038/s41586-018-0784-9

Chatterjee A, Seyfferth J, Lucci J, Gilsbach R, Preiss S, Bottinger L, Martensson CU, Panhale A, Stehle T, Kretz O, et al (2016) MOF acetyl transferase regulates transcription and respiration in mitochondria. Cell 167: 722-738.e3. doi:10.1016/j.cell.2016.09.052

Chiu AC, Suzuki HI, Wu X, Mahat DB, Kriz AJ, Sharp PA (2018) Transcriptional pause sites delineate stable nucleosome-associated premature polyadenylation suppressed by U1 snRNP. Mol Cell 69: 648-663.e7. doi:10.1016/j.molcel.2018.01.006

Chomczynski P, Sacchi N (2006) The single-step method of RNA isolation by acid guanidinium thiocyanate-phenol-chloroform extraction: Twenty-something years on. Nat Protoc 1: 581-585. doi:10.1038/ nprot.2006.83

Colby C, Morgan MJ (1971) Interferon induction and action. Annu Rev Microbiol 25: 333-360. doi:10.1146/annurev.mi.25.100171.002001

David M, Dzamba M, Lister D, Ilie L, Brudno M (2011) SHRiMP2: Sensitive yet practical SHort read mapping. Bioinformatics 27: 1011-1012. doi:10.1093/bioinformatics/btr046

Di Donato N, Neuhann T, Kahlert AK, Klink B, Hackmann K, Neuhann I, Novotna B, Schallner J, Krause C, Glass IA, et al (2016) Mutations in EXOSC2 are associated with a novel syndrome characterised by retinitis pigmentosa, progressive hearing loss, premature ageing, short stature, mild intellectual disability and distinctive gestalt. J Med Genet 53: 419-425. doi:10.1136/jmedgenet-2015-103511

Djafarzadeh S, Vuda M, Takala J, Ochs M, Jakob SM (2011) Toll-like receptor-3induced mitochondrial dysfunction in cultured human hepatocytes. Mitochondrion 11: 83-88. doi:10.1016/j.mito.2010.07.010

Dobin A, Davis CA, Schlesinger F, Drenkow J, Zaleski C, Jha S, Batut P, Chaisson M, Gingeras TR (2013) STAR: Ultrafast universal RNA-seq aligner. Bioinformatics 29: 15-21. doi:10.1093/bioinformatics/bts635 
Dou Z, Ghosh K, Vizioli MG, Zhu J, Sen P, Wangensteen KJ, Simithy J, Lan Y, Lin Y, Zhou Z, et al (2017) Cytoplasmic chromatin triggers inflammation in senescence and cancer. Nature 550: 402-406. doi:10.1038/ nature24050

Franchi L, Eigenbrod T, Munoz-Planillo R, Ozkurede U, Kim YG, Arindam C, Gale M Jr., Silverman RH, Colonna M, Akira S, et al (2014) Cytosolic doublestranded RNA activates the NLRP3 inflammasome via MAVS-induced membrane permeabilization and K+ efflux. J Immunol 193: 4214-4222. doi:10.4049/jimmunol.1400582

Fujita K, Mondal AM, Horikawa I, Nguyen GH, Kumamoto K, Sohn JJ, Bowman ED, Mathe EA, Schetter AJ, Pine SR, et al (2009) p53 isoforms Delta133p53 and p53beta are endogenous regulators of replicative cellular senescence. Nat Cell Biol 11: 1135-1142. doi:10.1038/ncb1928

Giannakakis A, Zhang J, Jenjaroenpun P, Nama S, Zainolabidin N, Aau MY, Yarmishyn AA, Vaz C, Ivshina AV, Grinchuk OV, et al (2015) Contrasting expression patterns of coding and noncoding parts of the human genome upon oxidative stress. Sci Rep 5: 9737. doi:10.1038/srep09737

Hernandez-Segura A, de Jong TV, Melov S, Guryev V, Campisi J, Demaria M (2017) Unmasking transcriptional heterogeneity in senescent cells. Curr Biol 27: 2652-2660.e4. doi:10.1016/j.cub.2017.07.033

Hung T, Pratt GA, Sundararaman B, Townsend MJ, Chaivorapol C, Bhangale T, Graham RR, Ortmann W, Criswell LA, Yeo GW, et al (2015) The Ro60 autoantigen binds endogenous retroelements and regulates inflammatory gene expression. Science 350: 455-459. doi:10.1126/ science.aac7442

Jeanblanc M, Ragu S, Gey C, Contrepois K, Courbeyrette R, Thuret J-Y, Mann C (2012) Parallel pathways in RAF-induced senescence and conditions for its reversion. Oncogene 31: 3072-3085. doi:10.1038/onc.2011.481

Katlinskaya YV, Katlinski KV, Yu Q, Ortiz A, Beiting DP, Brice A, Davar D, Sanders C, Kirkwood JM, Rui H, et al (2016) Suppression of type I interferon signaling overcomes oncogene-induced senescence and mediates melanoma development and progression. Cell Rep 15: 171-180. doi:10.1016/j.celrep.2016.03.006

Kuleshov MV, Jones MR, Rouillard AD, Fernandez NF, Duan Q, Wang Z, Koplev S, Jenkins SL, Jagodnik KM, Lachmann A, et al (2016) Enrichr: A comprehensive gene set enrichment analysis web server 2016 update. Nucleic Acids Res 44: W90-W97. doi:10.1093/nar/gkw377

Lau L, Porciuncula A, Yu A, Iwakura Y, David G (2019) Uncoupling the senescence-associated secretory phenotype from cell cycle exit via interleukin-1 inactivation unveils its protumorigenic role. Mol Cell Biol 39: e00586-18. doi:10.1128/mcb.00586-18

Lazorthes S, Vallot C, Briois S, Aguirrebengoa M, Thuret J-Y, St Laurent G, Rougeulle C, Kapranov P, Mann C, Trouche D, et al (2015) A vlincRNA participates in senescence maintenance by relieving H2AZ-mediated repression at the INK4 locus. Nat Commun 6: 5971. doi:10.1038/ ncomms7918

Lemay JF, Larochelle M, Marguerat S, Atkinson S, Bahler J, Bachand F (2014) The RNA exosome promotes transcription termination of backtracked RNA polymerase II. Nat Struct Mol Biol 21: 919-926. doi:10.1038/ nsmb.2893

Li H, Handsaker B, Wysoker A, Fennell T, Ruan J, Homer N, Marth G, Abecasis G, Durbin R1000 Genome Project Data Processing Subgroup, (2009) The sequence alignment/map format and SAMtools. Bioinformatics 25: 2078-2079. doi:10.1093/bioinformatics/btp352

Liao Y, Smyth GK, Shi W (2014) featureCounts: An efficient general purpose program for assigning sequence reads to genomic features. Bioinformatics 30: 923-930. doi:10.1093/bioinformatics/btt656

Love MI, Huber W, Anders S (2014) Moderated estimation of fold change and dispersion for RNA-seq data with DESeq2. Genome Biol 15: 550. doi:10.1186/s13059-014-0550-8

Miao ZF, Wu JH, Wang ZN, Zhao TT, Xu HM, Song YX, Xing YN, Huang JY, Zhang JY, Liu XY, et al (2016) Endoglin overexpression mediates gastric cancer peritoneal dissemination by inducing mesothelial cell senescence. Hum Pathol 51: 114-123. doi:10.1016/j.humpath.2015.12.023

Montes M, Lund AH (2016) Emerging roles of IncRNAs in senescence. FEBS J 283: 2414-2426. doi:10.1111/febs.13679

Muniz L, Deb MK, Aguirrebengoa M, Lazorthes S, Trouche D, Nicolas E (2017) Control of gene expression in senescence through transcriptional read-through of convergent protein-coding genes. Cell Rep 21: 2433-2446. doi:10.1016/j.celrep.2017.11.006

Nilson KA, Lawson CK, Mullen NJ, Ball CB, Spector BM, Meier JL, Price DH (2017) Oxidative stress rapidly stabilizes promoter-proximal paused Pol II across the human genome. Nucleic Acids Res 45: 11088-11105. doi:10.1093/nar/gkx724

Ogami K, Chen Y, Manley JL (2018) RNA surveillance by the nuclear RNA exosome: Mechanisms and significance. Noncoding RNA 4: 8. doi:10.3390/ncrna4010008

Preker P, Nielsen J, Kammler S, Lykke-Andersen S, Christensen MS, Mapendano CK, Schierup MH, Jensen TH (2008) RNA exosome depletion reveals transcription upstream of active human promoters. Science 322: 1851-1854. doi:10.1126/science.1164096

Ramirez F, Ryan DP, Gruning B, Bhardwaj V, Kilpert F, Richter AS, Heyne S, Dundar F, Manke T (2016) deepTools2: A next generation web server for deep-sequencing data analysis. Nucleic Acids Res 44: W160-W165. doi:10.1093/nar/gkw257

Robinson JT, Thorvaldsdóttir H, Winckler W, Guttman M, Lander ES, Getz G, Mesirov JP (2011) Integrative genomics viewer. Nat Biotechnol 29: 24-26. doi:10.1038/nbt0111-24

Rodier F, Campisi J (2011) Four faces of cellular senescence. J Cell Biol 192: 547-556. doi:10.1083/jcb.201009094

Salminen A, Kauppinen A, Kaarniranta K (2012) Emerging role of NF-kappaB signaling in the induction of senescence-associated secretory phenotype (SASP). Cell Signal 24: 835-845. doi:10.1016/ j.cellsig.2011.12.006

Schlackow M, Nojima T, Gomes T, Dhir A, Carmo-Fonseca M, Proudfoot NJ (2017) Distinctive patterns of transcription and RNA processing for human lincRNAs. Mol Cell 65: 25-38. doi:10.1016/ j.molcel.2016.11.029

Schonborn J, Oberstrass J, Breyel E, Tittgen J, Schumacher J, Lukacs N (1991) Monoclonal antibodies to double-stranded RNA as probes of RNA structure in crude nucleic acid extracts. Nucleic Acids Res 19: 2993-3000. doi:10.1093/nar/19.11.2993

Schottmann G, Picker-Minh S, Schwarz JM, Gill E, Rodenburg RJT, Stenzel W, Kaindl AM, Schuelke M (2017) Recessive mutation in EXOSC3 associates with mitochondrial dysfunction and pontocerebellar hypoplasia. Mitochondrion 37: 46-54. doi:10.1016/ j.mito.2017.06.007

Silverman RH (2007) Viral encounters with 2',5'-oligoadenylate synthetase and RNase $L$ during the interferon antiviral response. J Virol 81: 12720-12729. doi:10.1128/jvi.01471-07

Sudmant PH, Lee H, Dominguez D, Heiman M, Burge CB (2018) Widespread accumulation of ribosome-associated isolated $3^{\prime}$ UTRs in neuronal cell populations of the aging brain. Cell Rep 25: 2447-2456.e4. doi:10.1016/j.celrep.2018.10.094

Takahashi A, Loo TM, Okada R, Kamachi F, Watanabe Y, Wakita M, Watanabe S, Kawamoto S, Miyata K, Barber GN, et al (2018) Downregulation of cytoplasmic DNases is implicated in cytoplasmic DNA accumulation and SASP in senescent cells. Nat Commun 12: 1249. doi:10.1038/ S41467-018-03555-8

Tani H, Mizutani R, Salam KA, Tano K, ljiri K, Wakamatsu A, Isogai T, Suzuki Y, Akimitsu N (2012) Genome-wide determination of RNA stability reveals hundreds of short-lived noncoding transcripts in mammals. Genome Res 22: 947-956. doi:10.1101/gr.130559.111 
Tani H, Numajiri A, Aoki M, Umemura T, Nakazato T (2019) Short-lived long noncoding RNAs as surrogate indicators for chemical stress in HepG2 cells and their degradation by nuclear RNases. Sci Rep 9: 20299. doi:10.1038/s41598-019-56869-y

Tarallo V, Hirano Y, Gelfand BD, Dridi S, Kerur N, Kim Y, Cho WG, Kaneko H, Fowler BJ, Bogdanovich S, et al (2012) DICER1 loss and Alu RNA induce age-related macular degeneration via the NLRP3 inflammasome and MyD88. Cell 149: 847-859. doi:10.1016/j.cell.2012.03.036

Tripathi V, Shen Z, Chakraborty A, Giri S, Freier SM, Wu X, Zhang Y, Gorospe M, Prasanth SG, Lal A, et al (2013) Long noncoding RNA MALAT1 controls cell cycle progression by regulating the expression of oncogenic transcription factor B-MYB. PLoS Genet 9: e1003368. doi:10.1371/ journal.pgen.1003368

Villa T, Barucco M, Martin-Niclos MJ, Jacquier A, Libri D (2020) Degradation of non-coding RNAs promotes recycling of termination factors at sites of transcription. Cell Rep 32: 107942. doi:10.1016/j.celrep.2020.107942

Wang J, Geesman GJ, Hostikka SL, Atallah M, Blackwell B, Lee E, Cook PJ, Pasaniuc B, Shariat G, Halperin E, et al (2014) Inhibition of activated pericentromeric SINE/Alu repeat transcription in senescent human adult stem cells reinstates self-renewal. Cell Cycle 10: 3016-3030. doi:10.4161/cc.10.17.17543
Wang K, Wu D, Zhang H, Das A, Basu M, Malin J, Cao K, Hannenhalli S (2018) Comprehensive map of age-associated splicing changes across human tissues and their contributions to age-associated diseases. Sci Rep 8: 10929. doi:10.1038/s41598-018-29086-2

Xu CL, Sang B, Liu GZ, Li JM, Zhang XD, Liu LX, Thorne RF, Wu M (2020) SENEBLOC, a long non-coding RNA suppresses senescence via p53dependent and independent mechanisms. Nucleic Acids Res 48: 3089-3102. doi:10.1093/nar/gkaa063

Yoon JH, Abdelmohsen K, Kim J, Yang X, Martindale JL, Tominaga-Yamanaka K, White EJ, Orjalo AV, Rinn JL, Kreft SG, et al (2013) Scaffold function of long non-coding RNA HOTAIR in protein ubiquitination. Nat Commun 4: 2939. doi:10.1038/ncomms3939

Zhou R, Yazdi AS, Menu P, Tschopp J (2011) A role for mitochondria in NLRP3 inflammasome activation. Nature 469: 221-225. doi:10.1038/ nature09663

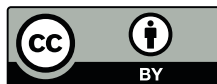

License: This article is available under a Creative Commons License (Attribution 4.0 International, as described at https://creativecommons.org/ licenses/by/4.0/). 